\title{
Full-Duplex MIMO in Cellular Networks: System-level Performance
}

\author{
Ratheesh K. Mungara, Member, IEEE, Ilaria Thibault, Member, IEEE, and \\ Angel Lozano, Fellow, IEEE
}

\begin{abstract}
This paper characterizes, through a stochastic geometry analysis, the increase in spectral efficiency that fullduplex transmission brings about in wireless networks. While, on isolated links, full-duplex promises a doubling of the spectral efficiency, in the context of a network this is weighted down by the corresponding rise in interference, and our characterization captures the balance of these effects. The analysis encompasses both the forward link (FL) and the reverse link (RL) with singleuser and multiuser transmissions. And, as a complement to the analysis, Monte-Carlo simulations on a Vodafone LTE field test network are also presented.

In the FL, the rise in interference is found to have minor impact and a doubling in spectral efficiency can indeed be approached, especially in microcellular networks. In the RL, however, a major difficulty arises in the form of exceedingly strong interference among base stations. This renders full-duplex transmission all but unfeasible in macrocellular networks (unless major countermeasures could be implemented) and undesirable in dense microcellular networks. Only in microcells with sufficient spacing among base stations does RL full-duplex pay off. Thus, full-duplex is seen not to blend easily with densification.
\end{abstract}

Index Terms-Full-duplex, half-duplex, cellular networks, stochastic geometry, interference, spectral efficiency, MIMO

\section{INTRODUCTION}

$\mathbf{S}$ ELF-INTERFERENCE cancellation, long unfeasible, is now becoming possible thanks to advanced combinations of analog and digital techniques [1]-[4]. Among the various self-interference cancellation architectures being proposed stands the one in [4], which provides the highest isolation (up to $110 \mathrm{~dB}$ ) between the transmit and receive chains for a single antenna. Although this technology is still in its infancy and challenges need to be addressed in relation to its form factors and robustness, it has the potential to enable many new applications in the wireless landscape. Examples are flexible RF filtering for easier and more agile spectrum management and simpler device front ends, self-backhauled

Manuscript received February 23, 2016; revised June 21, 2016 and December 19, 2016; accepted February 11, 2017. This work was supported in part by the Project TEC2015-66228-P (MINECO/FEDER, UE) and in part by the European Research Council under the H2020 Framework Programme/ERC under Grant 694974. The associate editor coordinating the review of this paper and approving it for publication was C.-B. Chae.

R. K. Mungara and A. Lozano are with the Department of Information and Communication Technologies, Universitat Pompeu Fabra (UPF), 08018 Barcelona, Spain. (e-mail: ratheesh.mungara@upf.edu, angel.lozano@upf.edu).

I. Thibault is with Vodafone Group Research and Development, Newbury, Berkshire, RG14 2PZ, U.K. (e-mail: ilaria.thibault@vodafone.com).

This work was supported by Project TEC2015-66228-P (MINECO/FEDER, UE) and by the European Research Council under the H2020 Framework Programme/ERC grant agreement 694974. relays that simplify network deployment, adjacent channel interference suppression for better coexistence, and higher resource utilization efficiency in cellular systems [5]-[7]. The latter refers to full-duplex wireless communication, which allows simultaneous transmission and reception on each timefrequency channel and which is the focus of this work. In addition to potentially doubling the spectral efficiency and reducing the end-to-end latency, full-duplex communication can offer solutions to problems such as hidden terminals, decoupling of FL (forward link) and RL (reverse link), and spectrum sharing [8]. Given the growing prevalence of multiantenna transmitters and receivers, it is desirable to further have full-duplex architectures featuring MIMO (multiple-input multiple-output), and indeed a MIMO full-duplex platform has already been prototyped [9].

By pushing the self-interference below the noise level, it is possible to achieve a near-doubling of spectral efficiency for an isolated link. However, this may not extrapolate to wireless networks where every transmitter in the system interferes with every receiver. The question then arises of whether, and by how much, full-duplex is beneficial over the standard halfduplex, and this question is precisely what motivates this paper.

Initial studies of this issue are presented in [10]-[15], where the average performance of full-duplex schemes has been characterized in wireless ad-hoc networks [10], [11], cellular networks [12]-[14], and heterogeneous networks [15]. Stochastic geometry is the toolbox invoked in all these studies, which allows for models that are both amenable to analysis and highly representative of the heterogeneous structure of emerging wireless networks.

In this work, we seek to further advance the understanding of the matter by incorporating aspects that were missing in those pioneering studies and that turn out to have major impact on the conclusions. A first such aspect relates to the pathloss exponent. Prior works [13] model the pathloss at a distance $r$ as $\beta r^{-\eta}$ where $\eta$ is the exponent while $\beta$ is the intercept, defined as the pathloss at a unit distance; this single-slope model is inadequate to represent the pathloss among BSs, which as we shall see is critical in full-duplex networks, and a multi-slope model is much more adequate. A second aspect that is ignored in prior works, because of the complexity that it brings into the analysis, is MIMO. In addition, most previous stochastic geometry analyses characterize network coverage or outage on the basis of the instantaneous signal-to-interference ratio (SIR). Nowadays, however, ergodic performance metrics obtained by expecting over the small-scale fading are 
operationally much more relevant because codewords span many fading realizations in frequency (because of the wide bandwidths), in space (because of the multiplicity of antennas) and in time (because of hybrid-ARQ) [16]. It is therefore more meaningful to focus on the local-average SIR and the ergodic spectral efficiency. This altogether motivates the analysis of full-duplex wireless networks that we present here, with the following contributions:

- We provide a unified stochastic geometry framework that encompasses both FL and RL in full-duplex cellular networks, with each BS serving multiple users on each time-frequency channel. In a preliminary version of this work [17], full-duplex performance was evaluated with single-antenna transceivers. In this paper, we extend the results in [17] to MIMO.

- By leveraging the analytical potency of stochastic geometry, we derive expressions for the system-wide distributions of local-average SIR and for the ergodic spectral efficiencies. We also obtain the spatially averaged spectral efficiencies in integral forms, which serve to gauge the system-level benefits. And, to complement the analysis, we conduct Monte-Carlo simulations on a Vodafone LTE field test network.

- We establish that, without serious additional interference management, full-duplex macrocells are not viable because of the strong interference among macro BSs.

- We show that, in microcells, full-duplex can yield close to the expected doubling in spectral efficiency in the FL. In the RL, and in absence of additional interference management, the BSs must be deployed with a certain minimum spacing in order to reap the benefits of fullduplex. This minimum spacing is considerable, and thus full-duplex is regretfully seen to be rather incompatible with densification.

The above observations are made under the premise of balanced traffic in the FL and RL, which is a reasonable assumption because of the increasing popularity of video uploading to social networking sites and recent growth in online storage services.

Notation: $\Gamma(\cdot)$ is the Gamma function, $\bar{\Gamma}(\cdot, \cdot)$ is the lower incomplete Gamma function, $E_{n}(\zeta)=\int_{1}^{\infty} \frac{e^{-\zeta t}}{t^{n}} \mathrm{~d} t$ is an exponential integral, $\mathcal{L}_{A}(\cdot)$ denotes the Laplace transform of $A$, and $\mathcal{N}_{\mathbb{C}}(\boldsymbol{a}, \boldsymbol{A})$ denotes a complex Gaussian random vector with mean $\boldsymbol{a}$ and variance matrix $\boldsymbol{A}$.

\section{NeTWORK MODEL}

We consider an interference-limited cellular network with both BSs and users supporting bidirectional communication, and conduct the analysis separately for FL and RL. Equipped with $N$ antennas, each BS communicates independent signal streams to $N$ single-antenna users. Each receiver has an estimate of the fading of its intended link(s), both in the FL and in the RL. Advantageously in full-duplex, because of channel reciprocity each BS can further utilize its RL fading estimates to serve its user(s) in the FL. Since the penalty caused by the application of pilot-assisted fading estimates in lieu of perfect estimates has been shown to be marginal [18], we assume the fading estimates to be perfect. An explicit account of the pilot overhead incurred to gather these estimates would equally affect half- and full-duplex systems, not having any bearing on the benefits of the latter over the former.

\section{A. Forward Link}

In the FL, the receiver under consideration is a user and the transmitter is the closest BS. The BS locations $\left\{b_{k}\right\}$ are modeled by a homogeneous Poisson point process (PPP), $\Phi_{\mathrm{b}} \subset \mathbb{R}^{2}$, with density $\lambda_{\mathrm{b}}$. If the users are associated with their closest BSs, then the user locations are dependent on their serving BS locations and violate the PPP condition from the typical-user viewpoint. Faced with this obstacle, we borrow a modeling assumption that is shown to be tight in [19] and whose validity for our purposes is examined later in the paper: the user locations $\left\{u_{j}\right\}$ belong to another independent homogeneous PPP $\Phi_{\mathrm{u}}$ with matched density, i.e., $\lambda_{\mathrm{u}}=N \lambda_{\mathrm{b}}$.

\section{B. Reverse Link}

In the RL, the receiver under consideration is a BS and the intended transmitters are the $N$ closest users. Borrowing techniques from [20], [21], the analysis could be extended to incorporate RL power control and relax the constraint that the intended transmitters be the closest users. While quantitatively very interesting, this extension is not expected to modify the qualitative conclusions because the performance of full-duplex in the RL is mainly limited by the strong interference from the BSs.

The aforementioned model can be viewed as a full-duplex network (cf. Fig. 1), where each BS simultaneously communicates with the strongest $N$ users in both FL and RL, yet the user distribution is equally favorable for both half-duplex and full-duplex.

To facilitate the readability of the equations, we place $\rightarrow$ and $\leftarrow$ markers atop the FL and RL variables, respectively.

\section{Signal ANd Propagation Models}

User antennas are unit-gain while BS antennas have a gain $G_{\mathrm{b}}$. We denote by $P_{\mathrm{b}}$ and $P_{\mathrm{u}}$ the transmit powers of BSs and users, respectively.

\section{A. Forward Link}

The isolation between the transmit and receive chains is not perfect. Depending on the hardware complexity, form factors at the transceivers, and various imperfections, there may be residual self-interference that acts as additional noise [4]. We introduce $\vartheta$ to model the residual self-interference as a function of the transmit-receive isolation, itself denoted by $\varepsilon$.

By Slivnyak's Theorem [22], we consider a receiving user at the origin and focus the analysis on its link, indexed by 0 . This link, whose user and serving BS are respectively located at $u_{0}$ (the origin) and $b_{0}$, serves as the typical link in the network (cf. Fig. 1a). The user at $u_{0}$ observes

$$
\vec{y}_{0}=\sqrt{\frac{P_{\mathrm{b}}}{N} G_{\mathrm{b}} \beta r_{u_{0}, b_{0}}^{-\eta}} \boldsymbol{h}_{b_{0}, u_{0}}^{*} \boldsymbol{V}_{b_{0}} \boldsymbol{s}_{b_{0}}+\vec{z}_{0}
$$




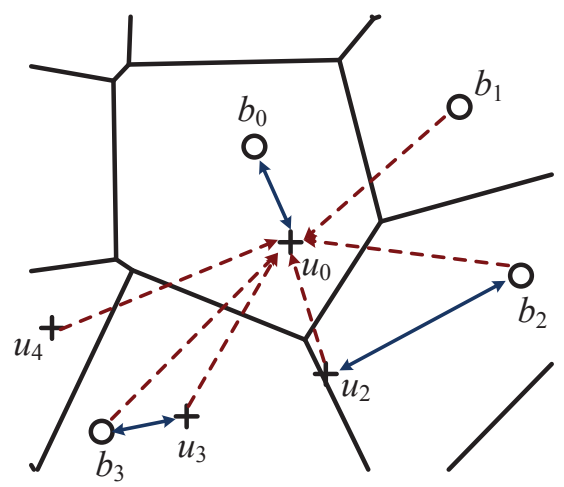

(a) Forward link

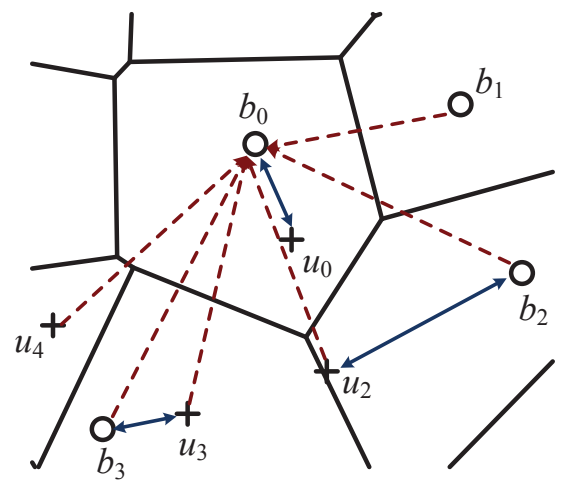

(b) Reverse link

Fig. 1: Full-duplex cellular network realization. In the FL, the receiver under consideration is a user (located at $u_{0}$ ) and the transmitter is the closest BS (located at $b_{0}$ ) while, in the RL, the receiver under consideration is a BS and the intended transmitter is the closest user. BS and user locations are indicated by $\circ$ and + markers, respectively. In this example, for illustrative purposes, $\lambda_{\mathrm{b}}=\lambda_{\mathrm{u}}$.

whose first term is the signal from its serving BS while the second term is the aggregate interference

$$
\begin{aligned}
\vec{z}_{0}= & \sum_{k=1}^{\infty} \sqrt{\frac{P_{\mathrm{b}}}{N} G_{\mathrm{b}} \beta r_{u_{0}, b_{k}}^{-\eta}} \boldsymbol{h}_{b_{k}, u_{0}}^{*} \boldsymbol{V}_{b_{k}} \boldsymbol{s}_{b_{k}} \\
& +\sum_{j=1}^{\infty} \sqrt{P_{\mathrm{u}} \beta_{\mathrm{u}} r_{u_{0}, u_{j}}^{-\eta_{\mathrm{u}}}} h_{u_{0}, u_{j}} s_{u_{j}}+\vartheta_{0}
\end{aligned}
$$

where the first summation spans the interference from other BSs, $\Phi_{\mathrm{b}} \backslash\left\{b_{0}\right\}$, and the second summation spans the interference from other users, $\Phi_{\mathrm{u}} \backslash\left\{u_{0}\right\}$, while $\vartheta_{0} \sim \mathcal{N}_{\mathbb{C}}\left(0, P_{\mathrm{u}} / \varepsilon\right)$ denotes the residual self-interference at the receiving user with transmit power $P_{\mathrm{u}}$ and transmit-receive isolation $\varepsilon$. In turn, $\beta$ and $\beta_{\mathrm{u}}$ are respectively the pathloss intercepts of BS-user and user-user links, $\eta$ and $\eta_{\mathrm{u}}$ are the corresponding pathloss exponents, $r_{u_{0}, b_{0}}$ is the distance from $b_{0}$ to $u_{0}, \boldsymbol{h}_{b_{0}, u_{0}} \in \mathbb{C}^{N \times 1}$ is the fading vector from $b_{0}$ to $u_{0}$ and $h_{u_{0}, u_{j}} \in \mathbb{C}$ is the fading coefficient from $u_{j}$ to $u_{0}$. The entries of $\boldsymbol{h}_{b_{0}, u_{0}}$ and $h_{u_{0}, u_{j}}$ are independent identically distributed (IID) samples drawn from $\mathcal{N}_{\mathbb{C}}(0,1)$. Meanwhile, $\boldsymbol{V}_{b_{0}} \in \mathbb{C}^{N \times N}$ is the precoder used by the BS at $b_{0}$ to transmit its data symbol vector $s_{b_{0}} \in \mathbb{C}^{N \times 1}$ while $s_{u_{j}} \in \mathbb{C}$ is the data symbol transmitted by the user at $u_{j}$. The entries of $s_{u_{0}}$ and $s_{b_{0}}$ are IID complex Gaussian such that $\mathbb{E}\left[\left|s_{u_{0}}\right|^{2}\right]=1$ and $\mathbb{E}\left[\boldsymbol{s}_{b_{0}} \boldsymbol{s}_{b_{0}}^{*}\right]=\boldsymbol{I}_{N}$. Power allocation is uniform across the $N$ signal streams, which gives $\mathbb{E}\left[\left\|\boldsymbol{V}_{b_{0}} \boldsymbol{s}_{b_{0}}\right\|_{2}^{2}\right]=N$.

Without loss of generality, BS and user locations are indexed in order of increasing distance, i.e., $r_{u_{0}, b_{k}}<r_{u_{0}, b_{k+1}}$ and $r_{u_{0}, u_{j}}<r_{u_{0}, u_{j+1}}$, while the BS at $b_{0}$ serves the users at $\left\{u_{n}\right\}_{n=0}^{N-1}$. Under these premises, the data symbol and precoder at $b_{0}$ can be written as $\boldsymbol{V}_{b_{0}}=\left[\boldsymbol{v}_{u_{0}, b_{0}}, \ldots, \boldsymbol{v}_{u_{N-1}, b_{0}}\right]$ and $\boldsymbol{s}_{b_{0}}=\left[s_{u_{0}, b_{0}}, \ldots, s_{u_{N-1}, b_{0}}\right]^{T}$ with $s_{u_{0}, b_{0}}$ the data symbol intended for the user at $u_{0}$ and $\boldsymbol{v}_{u_{0}, b_{0}}$ the corresponding precoder. With perfect CSI at the BS, the precoder $\boldsymbol{v}_{u_{0}, b_{0}}$ is designed according to the zero-forcing (ZF) strategy in order to prevent interference [23]. Mathematically, ZF means $\boldsymbol{h}_{b_{0}, u_{n}}^{*} \boldsymbol{v}_{u_{0}, b_{0}}=0$ for $n=1, \ldots, N-1$, which plugged into (1) gives

$$
\vec{y}_{0}=\sqrt{\frac{P_{\mathrm{b}}}{N} G_{\mathrm{b}} \beta r_{u_{0}, b_{0}}^{-\eta}} \boldsymbol{h}_{b_{0}, u_{0}}^{*} \boldsymbol{v}_{u_{0}, b_{0}} s_{u_{0}, b_{0}}+\vec{z}_{0} .
$$

\section{B. Reverse Link}

As mentioned earlier, we adopt a two-slope model for the BS-BS pathloss [24]. Denoting by $R_{\mathrm{c}}=4 \mathrm{~h}_{\mathrm{b}}^{2} / \lambda$ the so-called critical distance with $h_{b}$ the BS antenna height and $\lambda$ the wavelength, the BS-BS pathloss for a given distance $r_{b_{0}, b_{k}}$ is then

$$
\begin{cases}\beta_{\mathrm{b}} r_{b_{0}, b_{k}}^{-\eta_{\mathrm{b}}} & r_{b_{0}, b_{k}} \leq R_{\mathrm{c}} \\ \beta_{\mathrm{B}} r_{b_{0}, b_{k}}^{-\eta_{\mathrm{B}}} & r_{b_{0}, b_{k}}>R_{\mathrm{c}}\end{cases}
$$

where $\eta_{\mathrm{b}}$ and $\beta_{\mathrm{b}}$ are, respectively, the pathloss exponent and intercept of BS-BS links satisfying $r_{b_{0}, b_{k}} \leq R_{\mathrm{c}}$ while $\eta_{\mathrm{B}}$ and $\beta_{\mathrm{B}}$ are the pathloss exponent and intercept of BS-BS links satisfying $r_{b_{0}, b_{k}}>R_{\mathrm{c}}$. Unlike the users, the BSs may feature sophisticated hardware to suppress self-interference. Moreover, as we will demonstrate in Section VII, the RL performance is mainly limited by BS-BS interference and thus we can disregard the residual self-interference at the BSs. To analyze the RL, we shift the origin to the BS of interest, which observes (cf. Fig. 1b)

$$
\overleftarrow{\boldsymbol{y}}_{0}=\sum_{n=0}^{N-1} \sqrt{P_{\mathrm{u}} G_{\mathrm{b}} \beta r_{b_{0}, u_{n}}^{-\eta}} \boldsymbol{h}_{b_{0}, u_{n}} s_{u_{n}}+\overleftarrow{\boldsymbol{z}}_{0}
$$

whose first term is the signals from its intended users at $\left\{u_{n}\right\}_{n=0}^{N-1}$ while the second term is the aggregate interference

$$
\begin{aligned}
\overleftarrow{\boldsymbol{z}}_{0}= & \sum_{j=N}^{\infty} \sqrt{P_{\mathrm{u}} G_{\mathrm{b}} \beta r_{b_{0}, u_{j}}^{-\eta}} \boldsymbol{h}_{b_{0}, u_{j}} s_{u_{j}} \\
& +\sum_{k \in \mathcal{K}} \sqrt{\frac{P_{\mathrm{b}}}{N} G_{\mathrm{b}}^{2} \beta_{\mathrm{b}} r_{b_{0}, b_{k}}^{-\eta_{\mathrm{b}}}} \boldsymbol{H}_{b_{0}, b_{k}} \boldsymbol{V}_{b_{k}} \boldsymbol{s}_{b_{k}} \\
& +\sum_{k \notin \mathcal{K}} \sqrt{\frac{P_{\mathrm{b}}}{N} G_{\mathrm{b}}^{2} \beta_{\mathrm{B}} r_{b_{0}, b_{k}}^{-\eta_{\mathrm{B}}}} \boldsymbol{H}_{b_{0}, b_{k}} \boldsymbol{V}_{b_{k}} \boldsymbol{s}_{b_{k}}
\end{aligned}
$$

where the first summation spans the interference from other users, $\Phi_{\mathrm{u}} \backslash\left\{u_{n}\right\}_{n=0}^{N-1}$, and the second and third summations span the interference from other BSs, $\Phi_{\mathrm{b}} \backslash\left\{b_{0}\right\}$. In (6), $\mathcal{K}=$ $\left\{k: r_{b_{0}, b_{k}} \leq R_{\mathrm{c}}\right\}$ while $\boldsymbol{H}_{b_{0}, b_{k}} \in \mathbb{C}^{N \times N}$ is the fading matrix linking the BS at $b_{k}$ with the BS at $b_{0}$. The BS-BS fading can be modeled as Rayleigh, Rician or disregarded altogether [25]. 
As fading is seldom significant in BS-BS links, in this paper we set $\boldsymbol{H}_{b_{0}, b_{k}}=\boldsymbol{I}_{N}$ [26].

The BS at $b_{0}$ applies $\boldsymbol{W}_{b_{0}}=\left[\boldsymbol{w}_{b_{0}, u_{0}}, \ldots, \boldsymbol{w}_{b_{0}, u_{N-1}}\right] \in$ $\mathbb{C}^{N \times N}$ to process its received signal $\overleftarrow{\boldsymbol{y}}_{0}$, where $\boldsymbol{w}_{b_{0}, u_{n}}$ is the ZF filter corresponding to the signal from the user at $u_{n}$. At its output, $\boldsymbol{w}_{b_{0}, u_{n}}$ gives

$$
\boldsymbol{w}_{b_{0}, u_{n}}^{*} \overleftarrow{\boldsymbol{y}}_{0}=\sqrt{P_{\mathrm{u}} G_{\mathrm{b}} \beta r_{b_{0}, u_{n}}^{-\eta}} \boldsymbol{w}_{b_{0}, u_{n}}^{*} \boldsymbol{h}_{b_{0}, u_{n}} s_{u_{n}}+\boldsymbol{w}_{b_{0}, u_{n}}^{*} \overleftarrow{\boldsymbol{z}}_{0}
$$

Before proceeding with our analysis, we provide some distance distributions that are useful in subsequent derivations.

Given a homogeneous PPP $\Phi$ of intensity $\lambda$ with $r_{n}$ representing the distance from a point to its $(n+1)$ th neighbor, the probability density function (PDF) of $r_{n}$ is [27]

$$
f_{r_{n}}\left(r_{n}\right)=\frac{2(\pi \lambda)^{n+1}}{n !} r_{n}^{2 n+1} e^{-\pi \lambda r_{n}^{2}}
$$

and the joint PDF of $r_{n}$ and $r_{k}$ with $0 \leq n<k$ is [28]

$$
f_{r_{n}, r_{k}}\left(r_{n}, r_{k}\right)=\frac{4(\pi \lambda)^{k+1}}{(k-n-1) ! n !} \frac{r_{n}^{2 n+1} r_{k}}{\left(r_{k}^{2}-r_{n}^{2}\right)^{n-k+1}} e^{-\pi \lambda r_{k}^{2}}
$$

If $a_{n}$ denotes the ratio of the distances from a point to its $(n+$ 1 )th and $N$ th neighbors, i.e., $a_{n}=r_{n} / r_{N-1}$, then the joint PDF of $r_{n}$ and $a_{n}$ with $0 \leq n<N-1$ is (cf. Appendix A)

$$
\begin{aligned}
f_{r_{n}, a_{n}}\left(r_{n}, a_{n}\right)= & \frac{4(\pi \lambda)^{N}}{(N-n-2) ! n !} \frac{\left(1-a_{n}^{2}\right)^{N-n-2}}{a_{n}^{2 N-2 n-1}} \\
& \cdot r_{n}^{2 N-1} e^{-\frac{\pi \lambda r_{n}^{2}}{a_{n}^{2}}}
\end{aligned}
$$

\section{INTERFERENCE MODELING}

We follow the approach in [29]-[31] to model the cochannel interference terms $\vec{z}_{0}$ and $\overleftarrow{z}_{0}$. The local-average distributions of $\vec{z}_{0}$ and $\overleftarrow{z}_{0}$ are modeled as zero-mean complex Gaussian with respective matched conditional covariances $\mathbb{E}\left[\left|\vec{z}_{0}\right|^{2} \mid\left\{r_{u_{0}, b_{k}}, r_{u_{0}, u_{j}}\right\}\right]$ and $\mathbb{E}\left[\overleftarrow{\boldsymbol{z}}_{0} \overleftarrow{\boldsymbol{z}}_{0}^{*} \mid\left\{r_{b_{0}, u_{j}}, r_{b_{0}, b_{k}}\right\}\right]$ where the expectations are over the data and fading distributions.

In the FL, recalling (2), the conditional covariance of $\vec{z}_{0}$ for given interferer locations equals

$$
\begin{aligned}
\mathbb{E}\left[\left|\vec{z}_{0}\right|^{2} \mid\left\{r_{u_{0}, b_{k}}, r_{u_{0}, u_{j}}\right\}\right] & \frac{P_{\mathrm{b}} G_{\mathrm{b}} \beta}{N} \sum_{k=1}^{\infty} r_{u_{0}, b_{k}}^{-\eta} \mathbb{E}\left[\boldsymbol{h}_{b_{k}, u_{0}}^{*} \boldsymbol{V}_{b_{k}} \boldsymbol{s}_{b_{k}} \boldsymbol{s}_{b_{k}}^{*} \boldsymbol{V}_{b_{k}}^{*} \boldsymbol{h}_{b_{k}, u_{0}}\right] \\
& +P_{\mathrm{u}} \beta_{\mathrm{u}} \sum_{j=1}^{\infty} r_{u_{0}, u_{j}}^{-\eta_{\mathrm{u}}} \mathbb{E}\left[h_{u_{0}, u_{j}} s_{u_{j}} s_{u_{j}}^{*} h_{u_{0}, u_{j}}^{*}\right]+\mathbb{E}\left[\vartheta_{0} \vartheta_{0}^{*}\right] \\
= & \frac{P_{\mathrm{b}} G_{\mathrm{b}} \beta}{N} \sum_{k=1}^{\infty} r_{u_{0}, b_{k}}^{-\eta} \mathbb{E}\left[\left(\boldsymbol{h}_{b_{k}, u_{0}}^{*} \boldsymbol{V}_{b_{k}}\right)\left(\boldsymbol{h}_{b_{k}, u_{0}}^{*} \boldsymbol{V}_{b_{k}}\right)^{*}\right] \\
& +P_{\mathrm{u}} \beta_{\mathrm{u}} \sum_{j=1}^{\infty} r_{u_{0}, u_{j}}^{-\eta_{\mathrm{u}}} \mathbb{E}\left[h_{u_{0}, u_{j}} h_{u_{0}, u_{j}}^{*}\right]+\frac{P_{\mathrm{u}}}{\varepsilon} \\
= & P_{\mathrm{b}} G_{\mathrm{b}} \beta \sum_{k=1}^{\infty} r_{u_{0}, b_{k}}^{-\eta}+P_{\mathrm{u}} \beta_{\mathrm{u}} \sum_{j=1}^{\infty} r_{u_{0}, u_{j}}^{-\eta_{\mathrm{u}}}+\frac{P_{\mathrm{u}}}{\varepsilon}
\end{aligned}
$$

where (11) follows from the mutual independence of $\left\{s_{b_{k}}\right\}_{k=1}^{\infty}$ and $\left\{s_{u_{j}}\right\}_{j=1}^{\infty}$ while the first term in (13) follows from $\left(\boldsymbol{h}_{b_{k}, u_{0}}^{*} \boldsymbol{V}_{b_{k}}\right)$ being a $1 \times N$ vector with IID zero-mean unitvariance entries; thus, $\mathbb{E}\left[\left(\boldsymbol{h}_{b_{k}, u_{0}}^{*} \boldsymbol{V}_{b_{k}}\right)\left(\boldsymbol{h}_{b_{k}, u_{0}}^{*} \boldsymbol{V}_{b_{k}}\right)^{*}\right]=N$ [18].

Shifting to the RL and recalling (6), the conditional covariance of $\overleftarrow{z}_{0}$ for given interferer locations equals

$$
\begin{aligned}
& \mathbb{E}\left[\overleftarrow{\boldsymbol{z}}_{0} \overleftarrow{\boldsymbol{z}}_{0}^{*} \mid\left\{r_{b_{0}, u_{j}}, r_{b_{0}, b_{k}}\right\}\right] \\
& =P_{\mathrm{u}} G_{\mathrm{b}} \beta \sum_{j=N}^{\infty} r_{b_{0}, u_{j}}^{-\eta} \mathbb{E}\left[\boldsymbol{h}_{b_{0}, u_{j}} s_{u_{j}} s_{u_{j}}^{*} \boldsymbol{h}_{b_{0}, u_{j}}^{*}\right] \\
& +\frac{P_{\mathrm{b}} G_{\mathrm{b}}^{2} \beta_{\mathrm{b}}}{N} \sum_{k \in \mathcal{K}} r_{b_{0}, b_{k}}^{-\eta_{\mathrm{b}}} \mathbb{E}\left[\boldsymbol{V}_{b_{k}} \boldsymbol{s}_{b_{k}} \boldsymbol{s}_{b_{k}}^{*} \boldsymbol{V}_{b_{k}}^{*}\right] \\
& +\frac{P_{\mathrm{b}} G_{\mathrm{b}}^{2} \beta_{\mathrm{B}}}{N} \sum_{k \notin \mathcal{K}} r_{b_{0}, b_{k}}^{-\eta_{\mathrm{B}}} \mathbb{E}\left[\boldsymbol{V}_{b_{k}} \boldsymbol{s}_{b_{k}} \boldsymbol{s}_{b_{k}}^{*} \boldsymbol{V}_{b_{k}}^{*}\right] \\
& =P_{\mathrm{u}} G_{\mathrm{b}} \beta \sum_{j=N}^{\infty} r_{b_{0}, u_{j}}^{-\eta} \mathbb{E}\left[\boldsymbol{h}_{b_{0}, u_{j}} \boldsymbol{h}_{b_{0}, u_{j}}^{*}\right] \\
& +P_{\mathrm{b}} G_{\mathrm{b}}^{2} \beta_{\mathrm{b}} \sum_{k \in \mathcal{K}} r_{b_{0}, b_{k}}^{-\eta_{\mathrm{b}}} \boldsymbol{I}_{N}+P_{\mathrm{b}} G_{\mathrm{b}}^{2} \beta_{\mathrm{B}} \sum_{k \notin \mathcal{K}} r_{b_{0}, b_{k}}^{-\eta_{\mathrm{B}}} \boldsymbol{I}_{N} \\
& =\left[P_{\mathrm{u}} G_{\mathrm{b}} \beta \sum_{j=N}^{\infty} r_{b_{0}, u_{j}}^{-\eta}+P_{\mathrm{b}} G_{\mathrm{b}}^{2}\left(\beta_{\mathrm{b}} \sum_{k \in \mathcal{K}} r_{b_{0}, b_{k}}^{-\eta_{\mathrm{b}}}\right.\right. \\
& \left.\left.+\beta_{\mathrm{B}} \sum_{k \notin \mathcal{K}} r_{b_{0}, b_{k}}^{-\eta_{\mathrm{B}}}\right)\right] \boldsymbol{I}_{N}
\end{aligned}
$$

\section{SIR DistribUtions}

\section{A. Forward Link}

From (3) and (13), the instantaneous signal-to-interference ratio (SIR) experienced by the typical user in the FL is

$$
\begin{aligned}
\overrightarrow{S I R}_{0} & =\frac{\frac{P_{\mathrm{b}} G_{\mathrm{b}} \beta}{N} r_{u_{0}, b_{0}}^{-\eta} \mathbb{E}\left[\left|\boldsymbol{h}_{b_{0}, u_{0}}^{*} \boldsymbol{v}_{u_{0}, b_{0}} s_{u_{0}, b_{0}}\right|^{2} \mid\left\{\boldsymbol{h}_{b_{0}, u_{j}}\right\}_{j=0}^{N-1}\right]}{\mathbb{E}\left[\left|\vec{z}_{0}\right|^{2} \mid\left\{r_{u_{0}, b_{k}}, r_{u_{0}, u_{j}}\right\}\right]} \\
& =\frac{\frac{P_{\mathrm{b}} G_{\mathrm{b}} \beta}{N} r_{u_{0}, b_{0}}^{-\eta}\left|\boldsymbol{h}_{b_{0}, u_{0}}^{*} \boldsymbol{v}_{u_{0}, b_{0}}\right|^{2}}{P_{\mathrm{b}} G_{\mathrm{b}} \beta \sum_{k=1}^{\infty} r_{u_{0}, b_{k}}^{-\eta}+P_{\mathrm{u}} \beta_{\mathrm{u}} \sum_{j=1}^{\infty} r_{u_{0}, u_{j}}^{-\eta_{\mathrm{u}}}+\frac{P_{\mathrm{u}}}{\varepsilon}} \\
& =\vec{\rho}_{0}\left|\boldsymbol{h}_{b_{0}, u_{0}}^{*} \boldsymbol{v}_{u_{0}, b_{0}}\right|^{2}
\end{aligned}
$$

where the expectation in the numerator of (17) is over $s_{u_{0}, b_{0}}$, conditioned on the known fading (and therefore on the precoders), while

$$
\vec{\rho}_{0}=\frac{r_{u_{0}, b_{0}}^{-\eta}}{N \sum_{j=1}^{\infty} r_{u_{0}, u_{j}}^{-\eta_{\mathrm{u}}}+\mu_{\mathrm{u}} N \sum_{k=1}^{\infty} r_{u_{0}, b_{k}}^{-\eta}+\mu_{\mathrm{rsi}} N}
$$

is the local-average SIR at the typical user in the FL with

$$
\begin{aligned}
\mu_{\mathrm{u}} & =\frac{P_{\mathrm{u}} \beta_{\mathrm{u}}}{P_{\mathrm{b}} G_{\mathrm{b}} \beta} \\
\mu_{\mathrm{rsi}} & =\frac{P_{\mathrm{u}}}{P_{\mathrm{b}} G_{\mathrm{b}} \beta \varepsilon} .
\end{aligned}
$$

Since the $\boldsymbol{v}_{u_{0}, b_{0}}$ is independent of $\boldsymbol{h}_{b_{0}, u_{0}}$ in ZF precoding, the precoded channel $\boldsymbol{h}_{b_{0}, u_{0}}^{*} \boldsymbol{v}_{u_{0}, b_{0}} \sim \mathcal{N}_{\mathbb{C}}(0,1)$ and the power $\left|\boldsymbol{h}_{b_{0}, u_{0}}^{*} \boldsymbol{v}_{u_{0}, b_{0}}\right|^{2}$ is exponentially distributed with unit mean [18]. 


$$
\begin{array}{r}
F_{\vec{\rho}_{0} \mid r_{0}}(\gamma) \approx 1-\gamma \frac{e^{\frac{A}{2}}}{2^{L}} \sum_{\ell=0}^{L}\left(\begin{array}{c}
L \\
\ell
\end{array}\right) \sum_{m=0}^{M+\ell} \frac{(-1)^{m}}{D_{m}} \Re\left\{\frac { 1 } { t } \operatorname { e x p } \left(\pi \lambda_{\mathrm{b}} r_{0}^{2}-N \mu_{\mathrm{rsi}} r_{0}^{\eta} t+\frac{2 \pi \lambda_{\mathrm{b}}}{\eta} r_{0}^{2}(N t)^{\frac{2}{\eta}} \bar{\Gamma}\left(-\frac{2}{\eta}, N t\right)\right.\right. \\
\left.\left.-\pi N \lambda_{\mathrm{b}}\left(r_{0}^{\eta} N \mu_{\mathrm{u}} t\right)^{\frac{2}{\eta_{\mathrm{u}}}} \Gamma\left(1-\frac{2}{\eta_{\mathrm{u}}}\right)\right)\right\}
\end{array}
$$

TABLE I: Microcell network settings [26, Scenario 2]

\begin{tabular}{|c|c||c|c|}
\hline Parameter & Value & Parameter & Value \\
\hline \hline$P_{\mathrm{b}}$ & $24 \mathrm{dBm}$ & $\eta$ & 3.75 \\
\hline$P_{\mathrm{u}}$ & $23 \mathrm{dBm}$ & $\eta_{\mathrm{b}}, \eta_{\mathrm{B}}$ & 2,4 \\
\hline$G_{\mathrm{b}}$ & $5 \mathrm{dBi}$ & $\eta_{\mathrm{u}}$ & 4 \\
\hline$\beta$ & $-32.9 \mathrm{~dB}$ & $\mathrm{~h}_{\mathrm{b}}$ & $4 \mathrm{~m}$ \\
\hline$\beta_{\mathrm{b}}$ & $-38.45 \mathrm{~dB}$ & $\lambda$ & $0.15 \mathrm{~m}$ \\
\hline$\beta_{\mathrm{B}}$ & $-49.36 \mathrm{~dB}$ & $R_{\mathrm{c}}$ & $427 \mathrm{~m}$ \\
\hline$\beta_{\mathrm{u}}$ & $-55.78 \mathrm{~dB}$ & $\lambda_{\mathrm{b}}$ & $7.95 \mathrm{BSs} / \mathrm{km}^{2}$ \\
\hline
\end{tabular}

1) Local-Average SIR Distribution: The spatial distribution of the transmitter locations induces a distribution of its own for $\vec{\rho}_{0}$, which is derived next. As the exact distribution of the local-average aggregate interference generally does not admit a closed form, certain approximate characterizations have been proposed [32]-[35]. Here, we adapt the approach in [35]: obtain the distribution of $1 / \vec{\rho}_{0}$ exactly in the Laplace domain and then express the Laplace numerical inverse via Euler series expansion.

Proposition 1. For some given $r_{u_{0}, b_{0}}=r_{0}$, the cumulative distribution function $(C D F)$ of $\vec{\rho}_{0}$ is given by (23) in the next page, where

$$
t=\frac{(A+\mathrm{i} 2 \pi m) \gamma}{2}
$$

while $D_{0}=2$ and $D_{m}=1$ for $m \geq 1$. The parameters $A, L$ and $M$ control the accuracy, with suggested values for multiple-digit precision being $A=18.4, L=11$ and $M=$ 15 [36]. For our purposes, $A=9.21, L=5$ and $M=8$ yield a more-than-sufficient accuracy [37].

Proof. See Appendix B.

Eq. (23) can be uncoditioned via the density function in (8) (with $n=0$ and $\lambda=\lambda_{\mathrm{b}}$ ) to obtain

$$
F_{\vec{\rho}_{0}}(\gamma)=\int_{0}^{\infty} F_{\vec{\rho}_{0} \mid r_{0}}(\gamma) f_{r_{0}}\left(r_{0}\right) \mathrm{d} r_{0} .
$$

Example 1. Consider a network with single-antenna BSs of density $\lambda_{\mathrm{b}}=7.95 \mathrm{BSs} / \mathrm{km}^{2}$, which amounts to an average of one BS per circular cell of radius $200 \mathrm{~m}$, and with $\varepsilon=110$ $\mathrm{dB}$ [4] and typical values for the powers and the pathloss exponents (cf. Table I). Shown in Fig. 2 is a comparison of the CDFs $F_{\vec{\rho}_{0} \mid r_{0}=150}(\gamma)$ and $F_{\vec{\rho}_{0}}(\gamma)$ in (23) and (25), with $A=9.21, L=5$ and $M=8$, against their Monte-Carlo counterparts. An excellent match is observed, supporting the validity of the Euler series expansion of the inverse Laplace transform and the PPP assumption for the interfering user locations.

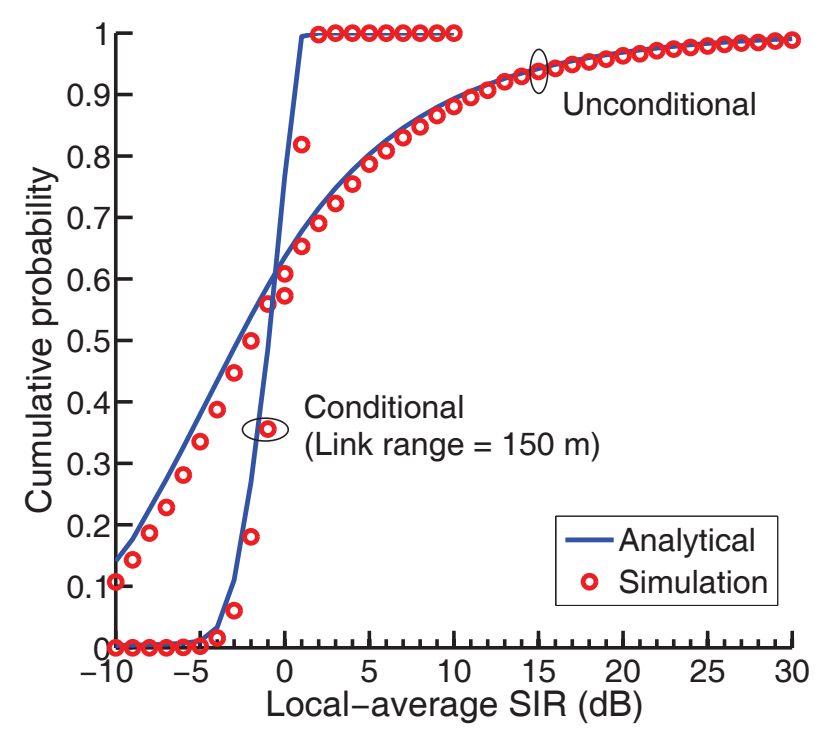

Fig. 2: CDFs of FL local-average SIR in a full-duplex microcellular network with single-antenna BSs and transmit-receive isolation $\varepsilon=110 \mathrm{~dB}$.

2) Instantaneous SIR Distribution: Given $\left\{r_{u_{0}, b_{k}}\right\}$ and $\left\{r_{u_{0}, u_{j}}\right\}$, the value of $\vec{\rho}_{0}$ in (20) becomes determined. Since the fading power $\left|\boldsymbol{h}_{b_{0}, u_{0}}^{*} \boldsymbol{v}_{u_{0}, b_{0}}\right|^{2}$ is exponentially distributed with unit mean, it follows from (19) that the FL SIR exhibits an exponential distribution with local-average $\vec{\rho}_{0}$ and hence its conditional $\mathrm{CDF}$ is

$$
F_{\overrightarrow{\operatorname{SIR}}_{0} \mid \vec{\rho}_{0}}(\gamma)=1-e^{-\gamma / \vec{\rho}_{0}}
$$

\section{B. Reverse Link}

Recalling (7), we can express the instantaneous SIR at the receiving $\mathrm{BS}$ corresponding to the $\mathrm{RL}$ signal stream from $u_{n}$ as

$$
\begin{aligned}
\overleftarrow{\mathrm{SIR}}_{0, n} & =\frac{P_{\mathrm{u}} G_{\mathrm{b}} \beta r_{b_{0}, u_{n}}^{-\eta} \mathbb{E}\left[\left|\boldsymbol{w}_{b_{0}, u_{n}}^{*} \boldsymbol{h}_{b_{0}, u_{n}} s_{u_{n}}\right|^{2} \mid\left\{\boldsymbol{h}_{b_{0}, u_{n}}\right\}_{n=0}^{N-1}\right]}{\boldsymbol{w}_{b_{0}, u_{n}}^{*} \mathbb{E}\left[\overleftarrow{\boldsymbol{z}}_{0} \overleftarrow{\boldsymbol{z}}_{0}^{*} \mid\left\{r_{b_{0}, u_{j}}, r_{b_{0}, b_{k}}\right\}\right] \boldsymbol{w}_{b_{0}, u_{n}}} \\
& =\overleftarrow{\rho}_{0, n}\left|\boldsymbol{w}_{b_{0}, u_{n}}^{*} \boldsymbol{h}_{b_{0}, u_{n}}\right|^{2}
\end{aligned}
$$

where the expectation in (27) is over $s_{u_{n}}$, conditioned on the known fading (and therefore on the receive filters), while

$$
\overleftarrow{\rho}_{0, n}=\frac{r_{b_{0}, u_{n}}^{-\eta}}{\sum_{j=N}^{\infty} r_{b_{0}, u_{j}}^{-\eta}+\mu_{\mathrm{b}} \sum_{k \in \mathcal{K}} r_{b_{0}, b_{k}}^{-\eta_{\mathrm{b}}}+\mu_{\mathrm{B}} \sum_{k \notin \mathcal{K}} r_{b_{0}, b_{k}}^{-\eta_{\mathrm{B}}}}
$$




$$
\begin{aligned}
& F_{\bar{\rho}_{0, n} \mid r_{n}, a_{n}}(\gamma) \approx 1-\gamma \frac{e^{\frac{A}{2}}}{2^{L}} \sum_{\ell=0}^{L}\left(\begin{array}{c}
L \\
\ell
\end{array}\right) \sum_{m=0}^{M+\ell} \frac{(-1)^{m}}{D_{m}} \Re\left\{\frac { 1 } { t } \operatorname { e x p } \left(\pi N \lambda_{\mathrm{b}} \frac{r_{n}^{2}}{a_{n}^{2}}+\pi \lambda_{\mathrm{b}} \kappa^{2} R_{\mathrm{c}}^{2}+\frac{2 \pi N \lambda_{\mathrm{b}}}{\eta} r_{n}^{2} t^{\frac{2}{\eta}} \bar{\Gamma}\left(-\frac{2}{\eta}, a_{n}^{\eta} t\right)\right.\right. \\
& \left.\left.+\frac{2 \pi \lambda_{\mathrm{b}}\left(r_{n}^{\eta} \mu_{\mathrm{B}} t\right)^{\frac{2}{\eta_{\mathrm{B}}}}}{\eta_{\mathrm{B}}} \bar{\Gamma}\left(-\frac{2}{\eta_{\mathrm{B}}}, \frac{r_{n}^{\eta} \mu_{\mathrm{B}}}{R_{\mathrm{c}}^{\eta_{\mathrm{B}}}} t\right)+\frac{2 \pi \lambda_{\mathrm{b}} R_{\mathrm{c}}^{2}}{\eta_{\mathrm{b}}}\left(E_{\frac{\eta_{\mathrm{b}}+2}{\eta_{\mathrm{b}}}}\left(\frac{r_{n}^{\eta} \mu_{\mathrm{b}}}{R_{\mathrm{c}}^{\eta_{\mathrm{b}}}} t\right)-\kappa^{2} E_{\frac{\eta_{\mathrm{b}}+2}{\eta_{\mathrm{b}}}}\left(\frac{r_{n}^{\eta} \mu_{\mathrm{b}}}{\left(\kappa R_{\mathrm{c}} \eta_{\mathrm{b}}\right.} t\right)\right)\right)\right\}
\end{aligned}
$$

is the RL local-average SIR at the typical BS corresponding to the signal stream from $u_{n}$ with

$$
\begin{aligned}
& \mu_{\mathrm{b}}=\frac{P_{\mathrm{b}} G_{\mathrm{b}} \beta_{\mathrm{b}}}{P_{\mathrm{u}} \beta} \\
& \mu_{\mathrm{B}}=\frac{P_{\mathrm{b}} G_{\mathrm{b}} \beta_{\mathrm{B}}}{P_{\mathrm{u}} \beta} .
\end{aligned}
$$

1) Local-Average SIR Distribution: Noting that BSs cannot be arbitrarily close in actual deployments, we introduce a parameter $0<\kappa \leq 1$ such that $r_{b_{0}, b_{1}}>\kappa R_{\mathrm{c}}$. We derive the CDF of $\overleftarrow{\rho}_{0}$ in terms of $\kappa$, thereby parameterizing the distribution by the guaranteed distance to the strongest interfering BS.

Proposition 2. For some given $r_{b_{0}, u_{n}}=r_{n}, r_{b_{0}, u_{N-1}}=r_{N-1}$ and $a_{n}=r_{b_{0}, u_{n}} / r_{b_{0}, u_{N-1}}$, the CDF of $\overleftarrow{\rho}_{0, n}$ is ${ }^{1}$ given by (32) in the next page, where $t, D_{m}, A, L$ and $M$ are as in Proposition 1.

Proof. See Appendix C.

The unconditional CDF of local-average SIR $F_{\bar{\rho}_{0, n}}(\gamma)$ can be computed as

$$
F_{\bar{\rho}_{0, n}}(\gamma)=\int_{0}^{1} \int_{0}^{\infty} F_{\bar{\rho}_{0, n} \mid r_{n}, a_{n}}(\gamma) f_{r_{n}, a_{n}}\left(r_{n}, a_{n}\right) \mathrm{d} r_{n} \mathrm{~d} a_{n}
$$

where $f_{r_{n}, a_{n}}(\cdot, \cdot)$ is the joint PDF of $r_{n}$ and $a_{n}$ corresponding to $\lambda=N \lambda_{\mathrm{b}}$ in (10) and, recall, the first integration variable $a_{n}$ (for $0 \leq n<N-1$ ) is the ratio of the distances from a point to its $(n+1)$ th and $N$ th neighbors, with $0<a_{n}<1$.

2) Instantaneous SIR Distribution: Given $\left\{r_{b_{0}, u_{j}}\right\}$ and $\left\{r_{b_{0}, b_{k}}\right\}$, the value of $\overleftarrow{\rho}_{0, n}$ in (29) becomes determined and it follows from (28) that

$$
F_{\overleftarrow{S I R}_{0, n} \mid \bar{\rho}_{0, n}}(\gamma)=1-e^{-\gamma / \bar{\rho}_{0, n}}
$$

\section{Spectral EFficiency}

\section{A. Forward Link}

1) Specific Network Geometry: For given $\vec{\rho}_{0}$, the FL ergodic link spectral efficiency (conditioned on $r_{b_{0}, u_{0}}=r_{0}$ ) is [38], [39]

$$
\begin{aligned}
\vec{C}\left(\vec{\rho}_{0} \mid r_{0}\right) & =\int_{0}^{\infty} \log _{2}(1+\gamma) \mathrm{d} F_{\overrightarrow{\operatorname{SR}}_{0} \mid \vec{\rho}_{0}, r_{0}}(\gamma) \\
& =e^{1 / \vec{\rho}_{0} \mid r_{0}} E_{1}\left(\frac{1}{\vec{\rho}_{0} \mid r_{0}}\right) \log _{2} e
\end{aligned}
$$

and its CDF equals

$$
F_{\vec{C} \mid r_{0}}(\gamma)=\mathbb{P}\left[e^{1 / \vec{\rho}_{0} \mid r_{0}} E_{1}\left(\frac{1}{\vec{\rho}_{0} \mid r_{0}}\right) \log _{2} e<\gamma\right]
$$

\footnotetext{
${ }^{1}$ One can derive the CDF directly in terms of $r_{n}$ and $r_{N-1}$ instead of $r_{n}$ and $a_{n}$, but the latter option yields a somewhat more compact expression.
}

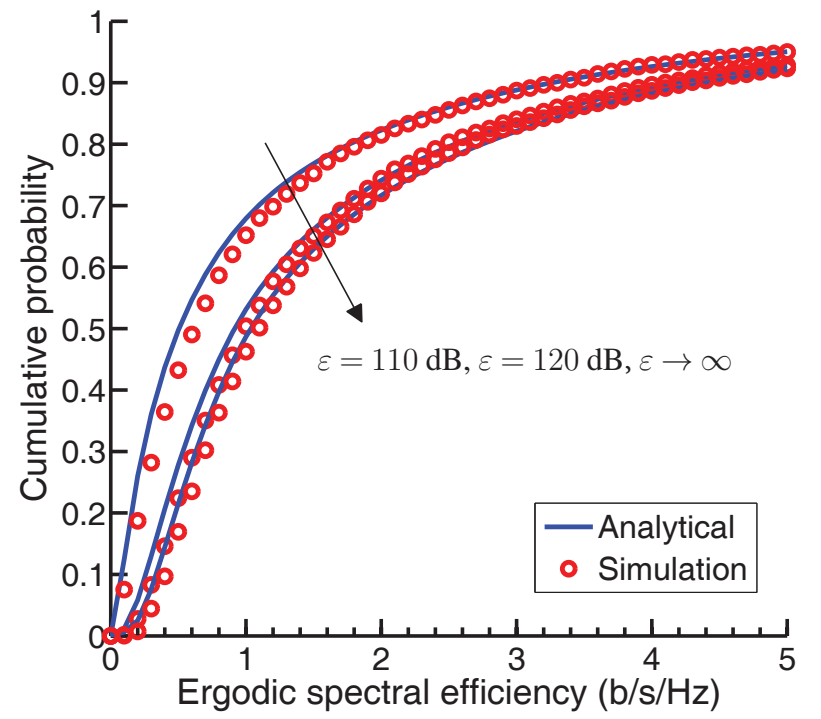

Fig. 3: CDFs of FL ergodic spectral efficiency in a full-duplex microcellular network with single-antenna BSs.

Invoking [40]

$$
e^{\nu} E_{1}(\nu) \log _{2} e \approx 1.4 \log \left(1+\frac{0.82}{\nu}\right)
$$

we can approximate (37) as

$$
F_{\vec{C} \mid r_{0}}(\gamma) \approx F_{\vec{\rho}_{0} \mid r_{0}}\left(\frac{e^{\frac{\gamma}{1.4}}-1}{0.82}\right),
$$

which is validated in the following example. Similarly, by leveraging the unconditional local-average SIR, $\vec{\rho}_{0}$, we can compute the corresponding ergodic link spectral efficiency as

$$
\vec{C}\left(\vec{\rho}_{0}\right)=e^{1 / \vec{\rho}_{0}} E_{1}\left(\frac{1}{\vec{\rho}_{0}}\right) \log _{2} e
$$

and its $\mathrm{CDF}$ as

$$
F_{\vec{C}}(\gamma) \approx F_{\overrightarrow{\rho_{0}}}\left(\frac{e^{\frac{\gamma}{1.4}}-1}{0.82}\right) .
$$

Example 2. For the same setting of Example 1, the approximate $\operatorname{CDF} F_{\vec{C}}(\cdot)$ is contrasted in Fig. 3, against its MonteCarlo counterpart for $\varepsilon=110 \mathrm{~dB}, \varepsilon=120 \mathrm{~dB}$ and $\varepsilon \rightarrow \infty$. Very good agreements are observed.

Recognizing that the performance gap between $\varepsilon=120 \mathrm{~dB}$ and $\varepsilon \rightarrow \infty$ is uniformly marginal, we assume $\varepsilon \rightarrow \infty$ or, equivalently, $\mu_{\mathrm{rsi}} \rightarrow 0$ in the remainder of the paper. 


$$
\overrightarrow{\bar{C}}=\int_{0}^{\infty} \frac{\log _{2} e}{\gamma+1} \int_{r_{0}>0}^{\infty} 2 \pi \lambda_{\mathrm{b}} r_{0} \exp \left(-N \mu_{\mathrm{rsi}} r_{0}^{\eta} \gamma+\frac{2 \pi \lambda_{\mathrm{b}} r_{0}^{2}(N \gamma)^{\frac{2}{\eta}}}{\eta} \bar{\Gamma}\left(\frac{-2}{\eta}, N \gamma\right)-\pi N \lambda_{\mathrm{b}}\left(r_{0}^{\eta} N \mu_{\mathrm{u}} \gamma\right)^{\frac{2}{\eta_{\mathrm{u}}}} \Gamma\left(1-\frac{2}{\eta_{\mathrm{u}}}\right)\right) \mathrm{d} r_{0} \mathrm{~d} \gamma
$$

2) Average Network Geometry: Next, we average the link spectral efficiency over all possible geometries.

Proposition 3. The average link spectral efficiency in the fullduplex cellular network model of Section II is given by (42).

\section{Proof. See Appendix D.}

Corollary 1. For $\eta_{\mathrm{u}}=\eta$ and $\mu_{\mathrm{rsi}} \rightarrow 0$, Proposition 3 reduces to

$$
\begin{aligned}
\overrightarrow{\vec{C}}= & \int_{0}^{\infty} \frac{\log _{2} e}{\gamma+1} \\
& \cdot \frac{1}{N\left(N \mu_{\mathrm{u}} \gamma\right)^{\frac{2}{\eta}} \Gamma\left(1-\frac{2}{\eta}\right)-\frac{2}{\eta}(N \gamma)^{\frac{2}{\eta}} \bar{\Gamma}\left(-\frac{2}{\eta}, N \gamma\right)} \mathrm{d} \gamma
\end{aligned}
$$

The users served by the BS at $b_{0}$ are located at $\left\{u_{n}\right\}_{n=0}^{N-1}$ and their individual ergodic link spectral efficiencies are identically distributed. Thus, the aggregate average spectral efficiency of the users served by the BS at $b_{0}$ equals $N \vec{C}$.

Example 3. For the parameters in Table I and $N=1$, the average spectral efficiency $\overrightarrow{\vec{C}}$, computed via (42) with $\mu_{\mathrm{rsi}} \rightarrow 0$, is $1.78 \mathrm{~b} / \mathrm{s} / \mathrm{Hz}$ while its simulated counterpart is $1.82 \mathrm{~b} / \mathrm{s} / \mathrm{Hz}$. The simulated result corresponds to the exact mutual information under the non-Gaussian interference in (2), evaluated through Monte-Carlo histograms and averaged over many fading realizations and interference locations. The match is excellent, supporting our interference modeling approach.

\section{B. Reverse Link}

1) Specific Network Geometry: Proceeding as in the FL, the RL ergodic spectral efficiencies of the typical BS (corresponding to the signal stream from $u_{n}$ ) for given $\overleftarrow{\rho}_{0, n}$ and $\overleftarrow{\rho}_{0, n} \mid r_{n}, a_{n}$ are

$$
\begin{gathered}
\overleftarrow{C}\left(\overleftarrow{\rho}_{0, n}\right)=e^{1 / \overleftarrow{\rho}_{0, n}} E_{1}\left(\frac{1}{\overleftarrow{\rho}_{0, n}}\right) \log _{2} e \\
\overleftarrow{C}\left(\overleftarrow{\rho}_{0, n} \mid r_{n}, a_{n}\right)=e^{1 / \overleftarrow{\rho}_{0, n} \mid r_{n}, a_{n}} E_{1}\left(\frac{1}{\overleftarrow{\rho}_{0, n} \mid r_{n}, a_{n}}\right) \log _{2} e
\end{gathered}
$$

with CDFs

$$
\begin{aligned}
F_{\bar{C}}(\gamma) & \approx F_{\bar{\rho}_{0, n}}\left(\frac{e^{\frac{\gamma}{1.4}}-1}{0.82}\right) \\
F_{\bar{C} \mid r_{n}, a_{n}}(\gamma) & \approx F_{\bar{\rho}_{0, n} \mid r_{n}, a_{n}}\left(\frac{e^{\frac{\gamma}{1.4}}-1}{0.82}\right) .
\end{aligned}
$$

\section{2) Average of all Network Geometries:}

Proposition 4. The average per-BS spectral efficiency in the $R L$ is given by (48) in the next page, where $f_{r_{n}, a_{n}}(\cdot)$ is the joint PDF of $r_{n}$ and $a_{n}$ corresponding to $\lambda=N \lambda_{\mathrm{b}}$ in (10).

Proof. See Appendix E.

For the last term in the summation (i.e., $n=N-1$ ), $a_{n}=1$ while $f_{r_{n}, a_{n}}(\cdot)$ reduces to $f_{r_{N-1}}(\cdot)$, the PDF of $r_{N-1}$ corresponding to $n=N-1$ and $\lambda=N \lambda_{\mathrm{b}}$ in (8), and the inner double integral reduces to a single integral.

For the single-antenna case, plugging $N=1$ in (48) we obtain the more compact expression as given in (49).

The derived expressions are not simple enough to provide immediate insight, but they are general and easy-to-evaluate using software packages such as Mathematica or MATLAB. For given system settings, $\overrightarrow{\vec{C}}$ and $\stackrel{\leftarrow}{C}$ can be solved for either instantaneously or within seconds (depending on whether $N=$ 1 or $N>1$ ); this is orders of magnitude faster than a MonteCarlo computation of the exact mutual information under nonGaussian interference.

\section{PeRformance of Full-Duplex Communication}

\section{A. Half-Duplex Baseline}

Unlike full-duplex, half-duplex communication utilizes separate time-frequency signaling channels for the FL and RL. Thus, the half-duplex spectral efficiency must be scaled by $1 / 2$ for a fair comparison with respect to full-duplex. The half-duplex counterparts to (40), (25) and (42) are then

$$
\begin{aligned}
\vec{C}^{\mathrm{HD}}\left(\vec{\rho}_{0}\right) & =\frac{1}{2} \lim _{\substack{\mu_{\mathrm{u}} \rightarrow 0 \\
\mu_{\mathrm{rsi}} \rightarrow 0}} \vec{C}\left(\vec{\rho}_{0}\right) \\
F_{\vec{C}^{\mathrm{HD}}}(\gamma) & \approx \lim _{\substack{\mu_{\mathrm{u}} \rightarrow 0 \\
\mu_{\mathrm{rsi}} \rightarrow 0}} F_{\vec{\rho}_{0}}\left(\frac{e^{\frac{\gamma}{0.7}}-1}{0.82}\right) \\
\overrightarrow{\vec{C}}^{\mathrm{HD}} & =\frac{1}{2} \lim _{\substack{\mu_{\mathrm{u}} \rightarrow 0 \\
\mu_{\mathrm{rsi}} \rightarrow 0}} \overrightarrow{\vec{C}}
\end{aligned}
$$

where $\mu_{\mathrm{u}} \rightarrow 0$ and $\mu_{\mathrm{rsi}} \rightarrow 0$ turn off the FL user transmissions. Plugging $\vec{C}(\cdot)$ into (52) and evaluating the integral by virtue of [41, Eq. 3.326.2], $\overrightarrow{\bar{C}}^{\mathrm{HD}}$ reduces to

$$
\overrightarrow{\vec{C}}^{\mathrm{HD}}=\frac{1}{2} \int_{0}^{\infty} \frac{-\eta \log _{2} e}{2(N \gamma)^{\frac{2}{\eta}}(\gamma+1) \bar{\Gamma}\left(-\frac{2}{\eta}, N \gamma\right)} \mathrm{d} \gamma
$$

Similarly, from (44), (33) and (48), we can recover for halfduplex

$$
\overleftarrow{C}^{\mathrm{HD}}\left(\overleftarrow{\rho}_{0, n}\right)=\frac{1}{2} \lim _{\substack{\mu_{\mathrm{b}} \rightarrow 0 \\ \mu_{\mathrm{B}} \rightarrow 0 \\ \kappa \rightarrow 0}} \overleftarrow{C}\left(\overleftarrow{\rho}_{0, n}\right)
$$




$$
\begin{aligned}
& \overleftarrow{\bar{C}}=\sum_{n=0}^{N-1} \int_{0}^{\infty} \frac{\log _{2} e}{\gamma+1} \int_{0}^{1} \int_{0}^{\infty} e^{\pi \lambda_{\mathrm{b}} \kappa^{2} R_{\mathrm{c}}^{2}+\frac{2 \pi \lambda_{\mathrm{b}} R_{\mathrm{c}}^{2}}{\eta_{\mathrm{b}}}}\left(E_{\frac{\eta_{\mathrm{b}}+2}{\eta_{\mathrm{b}}}}\left(\frac{r_{n}^{\eta}}{R_{\mathrm{c}}^{\eta} \mathrm{b}} \mu_{\mathrm{b}} \gamma\right)-\kappa^{2} E_{\frac{\eta_{\mathrm{b}}+2}{\eta_{\mathrm{b}}}}\left(\frac{r_{n}^{\eta}}{\left(\kappa R_{\mathrm{c}}\right)^{\eta_{\mathrm{b}}}} \mu_{\mathrm{b}} \gamma\right)\right) \\
& \cdot e^{\pi N \lambda_{\mathrm{b}} \frac{r_{n}^{2}}{a_{n}^{2}}+\frac{2 \pi N \lambda_{\mathrm{b}} r_{n}^{2} \gamma^{\frac{2}{\eta}}}{\eta} \bar{\Gamma}\left(\frac{-2}{\eta}, a_{n}^{\eta} \gamma\right)+\frac{2 \pi \lambda_{\mathrm{b}}\left(r_{n}^{\eta} \mu_{\mathrm{B}} \gamma\right)^{\frac{2}{\eta_{\mathrm{B}}}}}{\eta_{\mathrm{B}}} \bar{\Gamma}\left(\frac{-2}{\eta_{\mathrm{B}}}, \frac{r_{n}^{\eta}}{R_{\mathrm{C}}^{\eta} \mathrm{B}} \mu_{\mathrm{B}} \gamma\right)} f_{r_{n}, a_{n}}\left(r_{n}, a_{n}\right) \mathrm{d} r_{n} \mathrm{~d} a_{n} \mathrm{~d} \gamma
\end{aligned}
$$

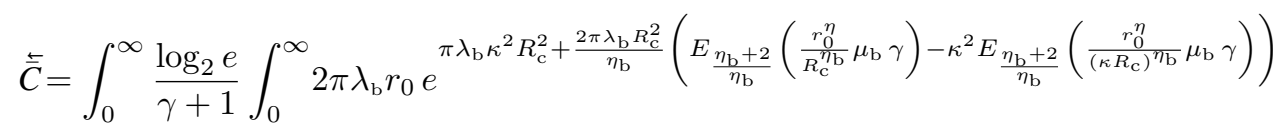

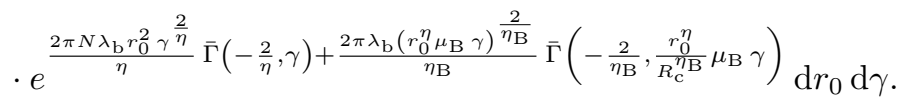

TABLE II: Macrocell network settings [26, Scenario 8]

\begin{tabular}{|c|c||c|c|}
\hline Parameter & Value & Parameter & Value \\
\hline \hline$P_{\mathrm{b}}$ & $46 \mathrm{dBm}$ & $\eta$ & 3.75 \\
\hline$P_{\mathrm{u}}$ & $23 \mathrm{dBm}$ & $\eta_{\mathrm{b}}$ & 2 \\
\hline$G_{\mathrm{b}}$ & $15 \mathrm{dBi}$ & $\eta_{\mathrm{B}}$ & 4 \\
\hline$\beta$ & $-15.3 \mathrm{~dB}$ & $\eta_{\mathrm{u}}$ & 4 \\
\hline$\beta_{\mathrm{b}}$ & $-38.45 \mathrm{~dB}$ & $\mathrm{~h}_{\mathrm{b}}$ & $20 \mathrm{~m}$ \\
\hline$\beta_{\mathrm{B}}$ & $1.0439 \mathrm{~dB}$ & $\lambda$ & $0.15 \mathrm{~m}$ \\
\hline$\beta_{\mathrm{u}}$ & $-55.78 \mathrm{~dB}$ & $R_{\mathrm{c}}$ & $10667 \mathrm{~m}$ \\
\hline
\end{tabular}

$$
\begin{aligned}
& F_{\overleftarrow{C}^{\mathrm{HD}}}(\gamma) \approx \lim _{\substack{\mu_{\mathrm{b}} \rightarrow 0 \\
\mu_{\mathrm{B}} \rightarrow 0 \\
\kappa \rightarrow 0}} F_{\bar{\rho}_{0, n}}\left(\frac{e^{\frac{\gamma}{0.7}}-1}{0.82}\right)
\end{aligned}
$$

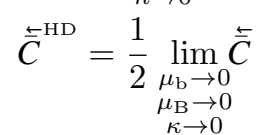

where $\mu_{\mathrm{b}} \rightarrow 0$ and $\mu_{\mathrm{B}} \rightarrow 0$ turn off the RL BS transmissions.

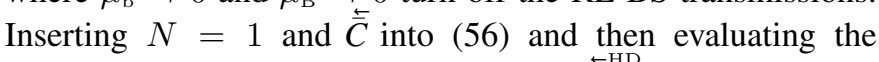
integrals by virtue of [41, Eq. 3.326.2], $\overleftarrow{C}^{\mathrm{HD}}$ is seen to equal

$$
\stackrel{\leftarrow}{\mathrm{HD}}^{\mathrm{HD}}=\frac{1}{2} \int_{0}^{\infty} \frac{-\eta \log _{2} e}{2 \gamma^{\frac{2}{\eta}}(\gamma+1) \bar{\Gamma}\left(-\frac{2}{\eta}, \gamma\right)} \mathrm{d} \gamma .
$$

\section{B. Performance Evaluation}

Armed with the full-duplex expressions derived in Sections V-VI, and with the half-duplex baselines just obtained, we can proceed to evaluate the performance advantage that full-duplex brings about when $\mu_{\mathrm{rsi}} \rightarrow 0$.

Example 4. Consider a macrocellular network with typical values for the powers and the pathloss exponents (cf. Table II). Single-antenna BSs with densities $\lambda_{\mathrm{b}}=1.27 \mathrm{BSs} / \mathrm{km}^{2}$ and $\lambda_{\mathrm{b}}=0.56 \mathrm{BSs} / \mathrm{km}^{2}$ are considered, respectively amounting to an average of one BS per circular cell of radii $500 \mathrm{~m}$ and $750 \mathrm{~m}$. The intended link distance is $r_{b_{0}, u_{0}}=100 \mathrm{~m}$ while $\kappa=0.1$ (equivalently, $r_{b_{0}, b_{1}}>1066.7 \mathrm{~m}$ ). Fig. 4 shows, parameterized by $\lambda_{\mathrm{b}}$, the CDF of $\overleftarrow{\rho}_{0}$ obtained by applying (32). The overwhelming interference among macro BSs - due to the low BS-BS pathloss for distances below $R_{\mathrm{c}}$-yields SIRs that are simply too low for viable full-duplex operation. Through

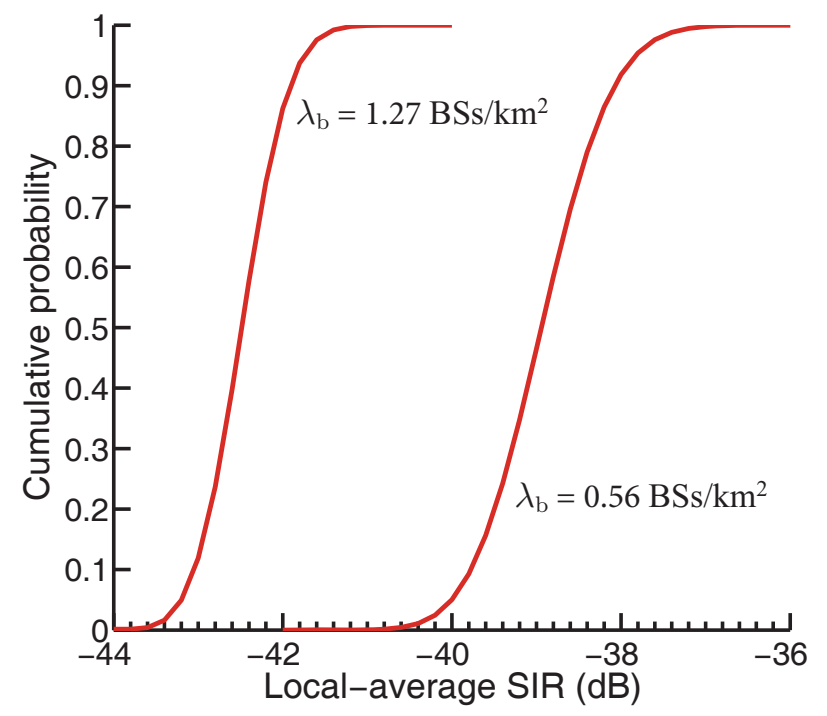

Fig. 4: CDFs of RL local-average SIR as function of $\lambda_{\mathrm{b}}$, for a full-duplex macrocell network with $r_{b_{0}, u_{0}}=100 \mathrm{~m}, N=1$ and $\kappa=0.1$ (equivalently, $\left.r_{b_{0}, b_{1}}>1066.7 \mathrm{~m}\right)$.

simulations, we have observed this observation to hold with sectorized antennas at the BSs [17].

Having exemplified how full-duplex is not feasible in macrocell RLs, at least not without additional interference management tailored to full-duplex, we next focus on microcells (cf. Table I).

Example 5. Consider a microcell network with single-antenna BSs, i.e., $N=1$ and $\lambda_{\mathrm{b}}=\lambda_{\mathrm{u}}$. Fig. 5 compares the FL ergodic spectral efficiency CDFs of full-duplex and half-duplex (cf. (41) and (51)). Full-duplex is superior to half-duplex in a vast majority of network situations and, as illustrated in the inset of Fig. 5, it achieves a spectral efficiency gain factor of 1.9 in $80 \%$ of cases. Then, Fig. 6 presents the same comparison for the RL, with two distinct values for $\kappa$. When $\kappa=3 / 4$, i.e., when the first interfering BS is within a critical distance of the receiving $\mathrm{BS}$, full-duplex is markedly inferior to half-duplex. However, for $\kappa=1$, full-duplex is uniformly 


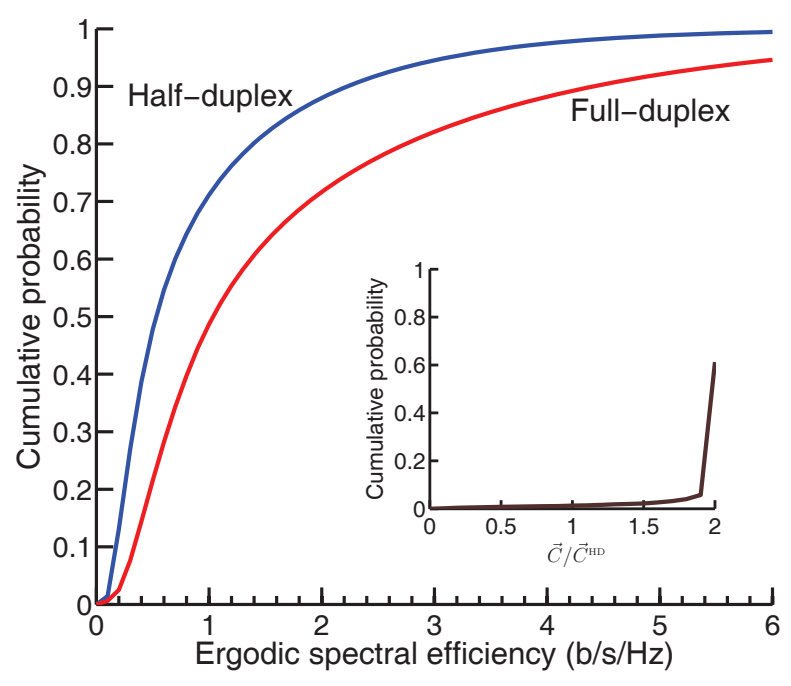

Fig. 5: Main plot: CDFs of FL ergodic spectral efficiency for a microcell network with half- and full-duplex. Inset: CDF of the ratio of the two spectral efficiencies. In both plots, single-antenna BSs are considered.

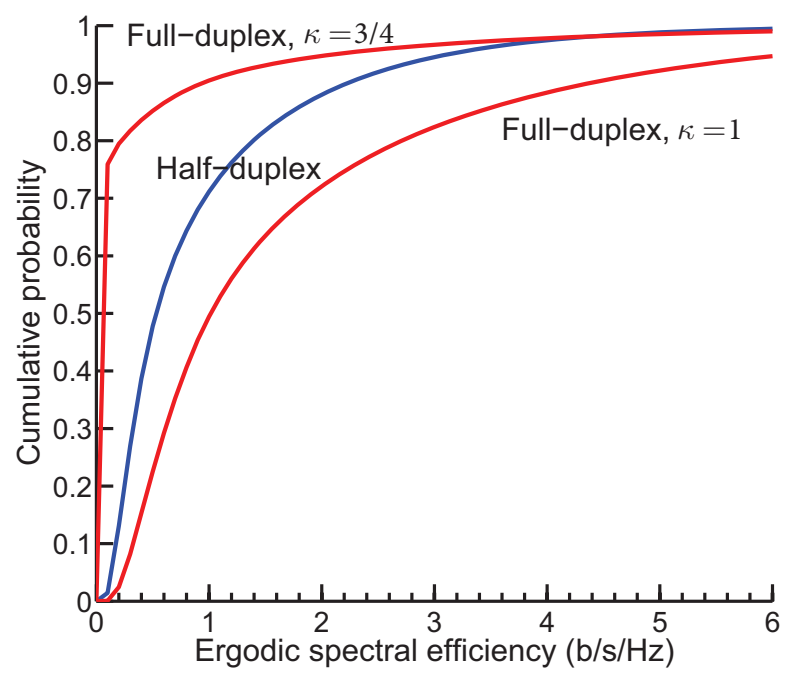

Fig. 6: CDF of RL ergodic spectral efficiency for a single-antenna microcell network with half-duplex and full-duplex.

superior to half-duplex, pointing to the need for a careful planning in full-duplex deployments.

Example 6. As a final step, we quantify the average benefits of full-duplex. For the microcell settings in Table I, the FL average spectral efficiencies (cf. (42) and (53)) for half-duplex and full-duplex are presented in Table III, where the gain factor due to full-duplex is seen to exceed 1.9 for varying $N$.

TABLE III: FL average spectral efficiency (b/s/Hz) for a microcell network with half-duplex and full-duplex

\begin{tabular}{|c|c|c|c|}
\hline$N$ & $N \vec{C}$ & $N \vec{C}^{\mathrm{HD}}$ & $\vec{C} / \vec{C}^{\mathrm{HD}}$ \\
\hline \hline 1 & 1.784 & 0.906 & 1.969 \\
\hline 2 & 2.657 & 1.350 & 1.968 \\
\hline 3 & 3.325 & 1.690 & 1.967 \\
\hline
\end{tabular}

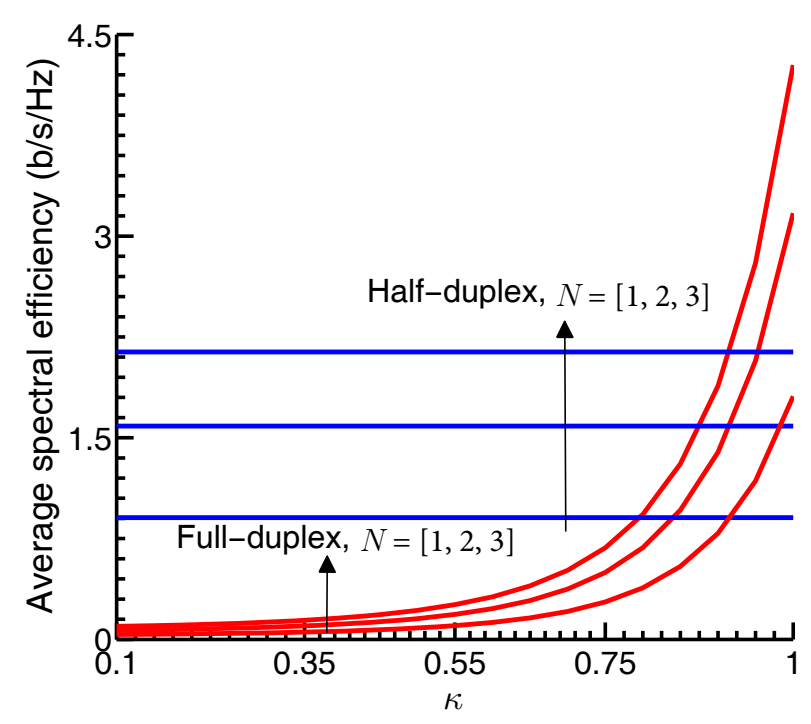

Fig. 7: RL average spectral efficiency for a microcell network with half-duplex and full-duplex.

The corresponding RL average spectral efficiencies (cf. (48) and (56)) are presented in Fig. 7, as a function of $\kappa$. The full-duplex average spectral efficiency increases with $\kappa$ and, at $\kappa=0.925$, it equals the value with half-duplex for $N=1$; thereafter, the gain increases rapidly. Hence, full-duplex outperforms half-duplex only if the BSs are apart by at least the critical distance. This is possible in microcell networks because of the relatively short critical distances (hundreds of meters).

Although a blanket utilization of full-duplex is not beneficial, there are situations (cf. Fig. 7) in which it is indeed advantageous. This points to a hybrid-duplex system that resorts to full-duplex or half-duplex, whichever is best, depending on the geometry. If the BS density is $\lambda_{\mathrm{b}}$, then the probability that the neighboring BS is apart by at least the critical distance equals $e^{-\pi \lambda_{\mathrm{b}} R_{\mathrm{c}}^{2}}$, and the fraction of BSs favorable for fullduplex is $e^{-\pi \lambda_{\mathrm{b}} R_{\mathrm{c}}^{2}}$. Based on this, the average system-level benefits of such a hybrid-duplex system can be quantified. Let us denote by $\overline{\mathcal{C}}^{\text {HD }}$ and $\overline{\mathcal{C}}^{\text {Hybrid }}$ the average area spectral efficiencies $\left(\mathrm{b} / \mathrm{s} / \mathrm{Hz} / \mathrm{km}^{2}\right)$ of half-duplex and hybrid-duplex, obtained by scaling the average link spectral efficiencies by the corresponding densities of BSs and users:

$$
\begin{aligned}
\overline{\mathcal{C}}^{\mathrm{HD}}= & \lambda_{\mathrm{u}} \overrightarrow{\vec{C}}^{\mathrm{HD}}+\lambda_{\mathrm{b}} \stackrel{\stackrel{\leftarrow}{\mathrm{HD}}}{\overline{\mathcal{C}}^{\mathrm{Hybrid}}=} e^{-\pi \lambda_{\mathrm{b}} R_{\mathrm{c}}^{2}}\left(\lambda_{\mathrm{u}} \overrightarrow{\vec{C}}+\lambda_{\mathrm{b}} \stackrel{\leftarrow}{\bar{C}}\right) \\
& +\left(1-e^{-\pi \lambda_{\mathrm{b}} R_{\mathrm{c}}^{2}}\right)\left(\lambda_{\mathrm{u}} \overrightarrow{\vec{C}}^{\mathrm{HD}}+\lambda_{\mathrm{b}} \stackrel{\stackrel{\leftarrow}{\mathrm{HD}}}{ }\right) .
\end{aligned}
$$

Example 7. Shown in Fig. 8 are the average area spectral efficiencies for half- and hybrid-duplex networks as a function of the BS density, for the microcell settings in Table I. The fraction of full-duplex BSs, $e^{-\pi \lambda_{\mathrm{b}} R_{\mathrm{c}}^{2}}$, decreases with the BS density $\lambda_{\mathrm{b}}$ and consequently the average area spectral efficiency of hybrid-duplex approaches the value of standalone half-duplex. This manifests again that full-duplex does not 


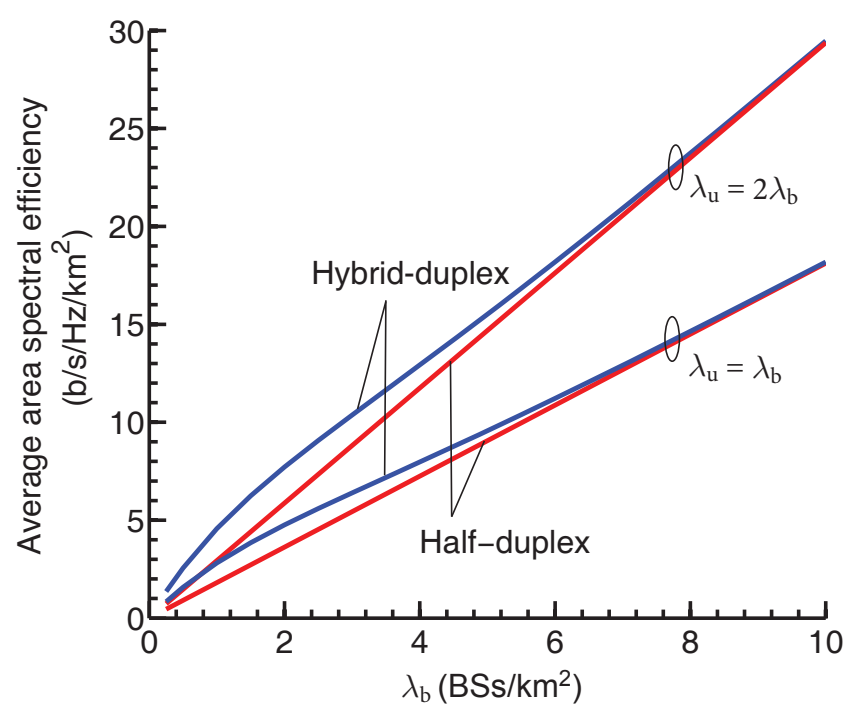

Fig. 8: Average area spectral efficiency for a microcell network with halfduplex and hybrid-duplex.

blend well with high densification.

As an alternative to gains in spectral efficiency, one can consider the sparsification in infrastructure density that fullduplex can bring about for a given area spectral efficiency (b/s/Hz per unit area). To achieve the same value thereof than a half-duplex network, only $\vec{C}^{\mathrm{HD}} / \overrightarrow{\vec{C}}$ as many BSs per unit area are needed under full-duplex.

\section{System-Level Benefits in a Vodafone Field Test Network}

To confirm the robustness of our PPP-based analytical findings, we further consider a Vodafone LTE field test network consisting of $64 \mathrm{BSs}$ over $1 \mathrm{~km}^{2}$ in the London area [42, Sec. III], obtain Monte-Carlo results for that network, and contrast them with our analysis. As the test network density is very high, the two-slope pathloss model is applied to BS-user and user-user links as well. Users are distributed such that the distance between each BS and its intended user is uniform within $[10,40] \mathrm{m}$. Heights of $4 \mathrm{~m}$ and $1.75 \mathrm{~m}$ are considered for the BS and user antenna, respectively, with typical 3GPP settings [26, Scenario 2].

Shown in Fig. 9 is a comparison of the FL ergodic spectral efficiency CDFs of full- and half-duplex. Consistent with our analysis, full-duplex is virtually always superior to half-duplex and, as illustrated in the inset of Fig. 9, it achieves a spectral efficiency gain factor of 1.7 in $50 \%$ of cases. Then, the same comparison for the RL is presented in Fig. 10. Again consistent with our analysis, full-duplex is markedly inferior to halfduplex due to the high density of BSs.

\section{SUMMARY}

The introduction of full-duplex transceivers in wireless networks would transform the interference landscape, foregoing long-standing interference barriers that have been in place since the inception of wireless communications, chiefly that BSs do not substantially interfere with other BSs. To assess the

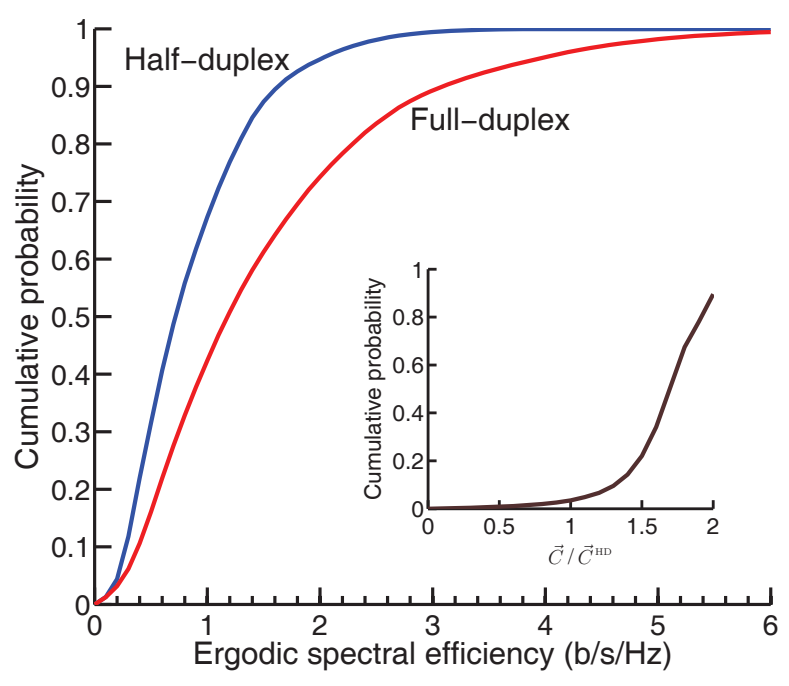

Fig. 9: Main plot: CDF of FL spectral efficiency for a Vodafone LTE field test network with half- and full-duplex. Inset: CDF of the ratio of the two spectral efficiencies. In both plots, single-antenna BSs are considered.

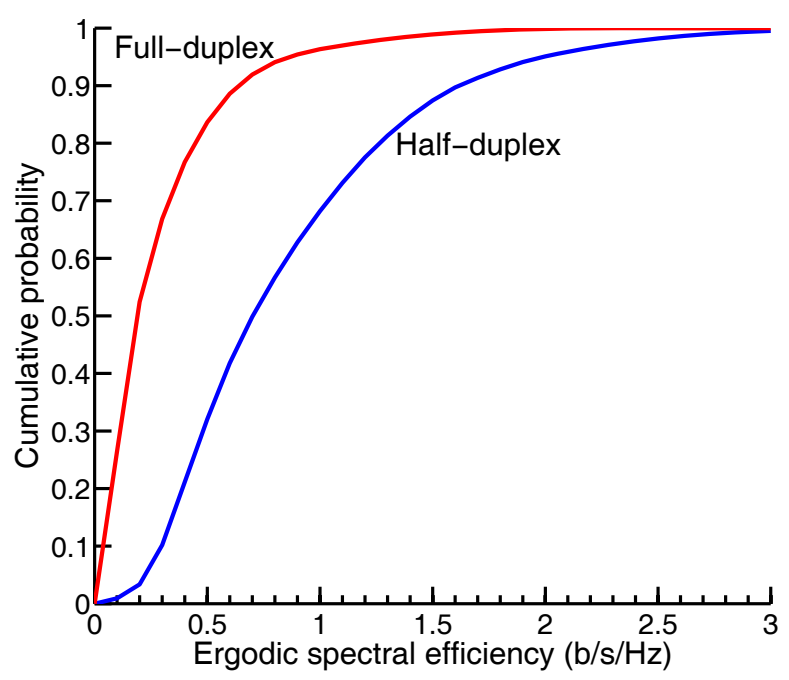

Fig. 10: CDF of RL spectral efficiency for a Vodafone LTE field test network with half and full-duplex.

system-level impact of introducing full-duplex transceivers, we have conducted a stochastic geometry analysis of a full-duplex cellular network and derived expressions for the system-wide distributions of local-average SIR and ergodic spectral efficiency. Variations of the formulation for both single-user and multiuser scenarios have been given. From these expressions, complemented by supporting examples and by simulations on a Vodafone LTE field network, we can conclude the following.

- Full-duplex RL is not viable in macrocells due to the excessive interference among BSs. In microcells, where the BS-BS pathloss is higher because of the lower elevations, full-duplex operation may be viable.

- Full-duplex consistently delivers a spectral efficiency gain factor of 1.9 in the FL of microcell networks. In the corresponding RL, however, full-duplex outperforms half-duplex only if the BSs are apart by at least the critical 
distance. Thus, full-duplex is (in principle, more on this below) at odds with network densification.

Altogether then, and insofar as no additional BS-BS or useruser interference management is introduced, our findings on the potential of full-duplex communication for $5 \mathrm{G}$ wireless systems are mostly negative. Further work would be welcome to solidify or tone down these conclusions, and we outline several directions of interest.

- The lower tail of the performance distribution of fullduplex in the FL is curbed by user-user interference. Given the availability of multiple channels, users could be parsed onto various sets in ways that avoided excessive interference, and indeed schemes that extend the classical notion of dynamic channel allocation [43] have been formulated in the context of device-to-device communication [44]-[48].

- In the RL, BS-BS interference limits the performance. This issue is well studied in the context of dynamic time-division duplexing [26], but only for distant BSs. It remains to be seen whether potential solutions such as null forming in elevation [49], cloud radio-access network processing [50], or interference alignment [29], [51] could achieve sufficient interference suppression. Advantageously, BS-BS links are very stable, hardly subject to fading, but according to our analysis several tens of $\mathrm{dB}$ of suppression might be necessary.

- Full-duplex improvements in spectral efficiency come at the expense of increased power consumption due to the sophisticated self-interference cancellation circuits [9]. It is thus worth studying full-duplex operation in the context of energy efficiency, a key desideratum for 5G [52], [53].

- BS antenna patterns in the elevation domain may alleviate the BS-BS interference (probably only slightly because their effect on nearby BSs is minor). Quantifying this improvement would be another interesting refinement of the present work.

- The balance between FL and RL traffic, which is immaterial under half-duplex because the corresponding signals do not interfere, becomes relevant with full-duplex. While in the present work we have considered balanced traffic, studying the impact of imbalances would be yet another relevant extension.

For $N>1$, an interesting variation of the full-duplex architecture considered in our analysis is one where only BSs are full-duplex, while users are half-duplex. Each BS then receives signals from a subset of users while transmitting to another (disjoint) subset. This variation is attractive from a hardware and power consumption vantage, as it eliminates the need for self-interference cancellation at the user devices (where form factors and power are key aspects), and it relaxes the issue of user-user interference. The BS-BS interference, however, is unaffected and thus the bulk of our conclusions continue to apply.

\section{Appendix A}

PROOF OF (10)

Utilizing the PDF $f_{r_{n}, r_{k}}(\cdot, \cdot)$ in (9) corresponding to $k=$ $N-1$, the joint PDF of $r_{n}$ and $a_{n}=r_{n} / r_{N-1}$ can be computed as

$$
\begin{aligned}
f_{r_{n}, a_{n}}\left(r_{n}, a_{n}\right)= & f_{r_{n}, r_{N-1}}\left(r_{n}, r_{N-1}\right)\left|\frac{\partial\left(r_{n}, r_{N-1}\right)}{\partial\left(r_{n}, a_{n}\right)}\right| \\
= & \frac{4(\pi \lambda)^{N}}{(N-n-2) ! n !}\left(\frac{r_{n}^{2}}{a_{n}^{2}}-r_{n}^{2}\right)^{N-n-2} \\
& \cdot \frac{r_{n}^{2 n+3}}{a_{n}^{3}} e^{-\frac{\pi \lambda r_{n}^{2}}{a_{n}^{2}}}
\end{aligned}
$$

which reduces to (10) after further simplification.

\section{APPENDIX B}

\section{PROOF OF PROPOSITION 1}

The distribution of $\vec{\rho}_{0}$ can be computed over the spatial locations of all interferers in the network. The CDF of $\vec{\rho}_{0}$ can be expressed as

$$
\begin{aligned}
F_{\overrightarrow{\rho_{0}}}(\gamma) & =\mathbb{P}\left[\vec{\rho}_{0} \leq \gamma\right] \\
& =\mathbb{P}\left(\frac{1}{\overrightarrow{\rho_{0}}} \geq 1 / \gamma\right) \\
& =1-F_{\frac{1}{\bar{\rho}_{0}}}(1 / \gamma) .
\end{aligned}
$$

The closed-form solution of (64) is unwieldy in general. Alternatively, we rely on the numerical inversion of the Laplace transform of $F_{1 / \vec{\rho}_{0}}(\cdot)$, which yields an accurate approximation in a series form for $F_{\vec{\rho}_{0}}(\gamma)$ [35].

$$
F_{\vec{\rho}_{0}}(\gamma)=1-\gamma \frac{e^{\frac{A}{2}}}{2^{L}} \sum_{\ell=0}^{L}\left(\begin{array}{c}
L \\
\ell
\end{array}\right) \sum_{m=0}^{M+b} \frac{(-1)^{m}}{D_{m}} \Re\left\{\mathcal{L}_{F_{1 / \vec{\rho}_{0}}}(t)\right\}
$$

where $t=\frac{(A+\mathrm{i} 2 \pi m) \gamma}{2}$ while $D_{0}=2$ and $D_{m}=1$ for $m \geq 1$. By utilizing the relation

$$
\mathcal{L}_{F_{1 / \vec{\rho}_{0}}}(t)=\frac{\mathcal{L}_{1 / \vec{\rho}_{0}}(t)}{t}
$$

we can rewrite (65) as

$$
F_{\vec{\rho}_{0}}(\gamma)=1-\gamma \frac{e^{\frac{A}{2}}}{2^{L}} \sum_{\ell=0}^{L}\left(\begin{array}{c}
L \\
\ell
\end{array}\right) \sum_{m=0}^{M+b} \frac{(-1)^{m}}{D_{m}} \Re\left\{\frac{\mathcal{L}_{1 / \vec{\rho}_{0}}(t)}{t}\right\} .
$$

Conditioned on $r_{u_{0}, b_{0}}=r_{0}$, the Laplace transform of $1 / \vec{\rho}_{0}$ is derived as

$$
\begin{aligned}
& \mathcal{L}_{1 / \vec{\rho}_{0} \mid r_{0}}(t) \\
& =\mathbb{E}\left[e^{-t / \vec{\rho}_{0}} \mid r_{0}\right] \\
& =\mathbb{E}\left[\operatorname { e x p } \left(-N \sum_{k=1}^{\infty} \frac{r_{u_{0}, b_{k}}^{-\eta}}{r_{0}^{-\eta} t}\right.\right. \\
& \left.\left.-N \mu_{\mathrm{u}} \sum_{j=1}^{\infty} \frac{r_{u_{0}, u_{j}}^{-\eta_{\mathrm{u}}}}{r_{0}^{-\eta}} t-\frac{N \mu_{\mathrm{rsi}}}{r_{0}^{-\eta}} t\right) \mid r_{0}\right] \\
& =e^{-\frac{N \mu_{\mathrm{rsi}} t}{r_{0}^{-\eta}}} \mathbb{E}_{\Phi_{\mathrm{b}}}\left[\prod_{k=1}^{\infty} \exp \left(-\frac{r_{u_{0}, b_{k}}^{-\eta}}{r_{0}^{-\eta}} N t\right) \mid r_{0}\right] \\
& \cdot \mathbb{E}_{\Phi_{\mathrm{u}}}\left[\prod_{j=1}^{\infty} \exp \left(-\frac{r_{u_{0}, u_{j}}^{-\eta_{\mathrm{u}}}}{r_{0}^{-\eta}} N \mu_{\mathrm{u}} t\right) \mid r_{0}\right]
\end{aligned}
$$




$$
\begin{aligned}
= & \exp \left(-N \mu_{\mathrm{rsi}} r_{0}^{\eta} t\right) \\
& \cdot \exp \left(-2 \pi \lambda_{\mathrm{b}} \int_{r_{0}}^{\infty}\left(1-e^{-r^{-\eta} r_{0}^{\eta} N t}\right) r \mathrm{~d} r\right) \\
& \cdot \exp \left(-2 \pi \lambda_{\mathrm{u}} \int_{0}^{\infty}\left(1-e^{-r^{-\eta_{\mathrm{u}}} r_{0}^{\eta} N \mu_{\mathrm{u}} t}\right) r \mathrm{~d} r\right) \\
= & \exp \left(-N \mu_{\mathrm{rsi}} r_{0}^{\eta} t\right) \\
& \cdot \exp \left(\pi \lambda_{\mathrm{b}} r_{0}^{2}+\frac{2 \pi \lambda_{\mathrm{b}} r_{0}^{2}(N t)^{\frac{2}{\eta}}}{\eta} \bar{\Gamma}\left(\frac{-2}{\eta}, N t\right)\right) \\
& \cdot \exp \left(-\pi N \lambda_{\mathrm{b}}\left(r_{0}^{\eta} N \mu_{\mathrm{u}} t\right)^{\frac{2}{\eta_{\mathrm{u}}}} \Gamma\left(1-\frac{2}{\eta_{\mathrm{u}}}\right)\right)
\end{aligned}
$$

where (68) follows by invoking the definition $\mathcal{L}_{F_{1 / \vec{p}_{0}}}$ and then evaluating integration by parts, (69) follows from substituting (20) in (68), (70) follows from the fact that the locations of BSs and users are two independent PPPs, (71) follows by separately invoking the definition of the probability generating functional (PGFL) of the PPP [22] for BS and user processes $\Phi_{\mathrm{b}}$ and $\Phi_{\mathrm{u}}$, and (72) follows from variable changes $t^{\prime}=N r^{-\eta} r_{0}^{\eta} t$ and $t^{\prime \prime}=r^{-\eta_{\mathrm{u}}} r_{0}^{\eta} N \mu_{\mathrm{u}} t$ and the relation $\lambda_{\mathrm{u}}=N \lambda_{\mathrm{b}}$. Invoking (72) into (67), we obtain the conditional $\mathrm{CDF} F_{\vec{\rho}_{0} \mid r_{0}}(\cdot)$ as in (23).

\section{APPENDIX C}

\section{PROOF OF PROPOSITION 2}

By leveraging the derivation of its FL counterpart, conditioned on $r_{b_{0}, u_{n}}=r_{n}, r_{b_{0}, u_{N-1}}=r_{N-1}$ and $a_{n}=r_{n} / r_{N-1}$, the CDF of the RL local-average SIR can be expressed as

$$
\begin{aligned}
& F_{\bar{\rho}_{0, n} \mid r_{n}, a_{n}}(\gamma) \\
& \quad=1-\gamma \frac{e^{\frac{A}{2}}}{2^{L}} \sum_{\ell=0}^{L}\left(\begin{array}{c}
L \\
\ell
\end{array}\right) \sum_{m=0}^{M+b} \frac{(-1)^{m}}{D_{m}} \Re\left\{\frac{\mathcal{L}_{1 / \bar{\rho}_{0, n} \mid r_{n}, a_{n}}(t)}{t}\right\}
\end{aligned}
$$

and the Laplace transform $\mathcal{L}_{1 / \bar{\rho}_{0, n} \mid r_{n}, a_{n}}(t)$ is derived as

$$
\begin{aligned}
& \mathcal{L}_{1 / \bar{\rho}_{0, n} \mid r_{n}, a_{n}}(t) \\
& =\mathbb{E}\left[e^{-t / \bar{\rho}_{0, n}} \mid r_{n}, a_{n}\right] \\
& =\mathbb{E}\left[\operatorname { e x p } \left(-\sum_{j=N}^{\infty} \frac{r_{b_{0}, u_{j}}^{-\eta}}{r_{n}^{-\eta}} t-\sum_{k \in \mathcal{K}} \frac{r_{b_{0}, b_{k}}^{-\eta_{\mathrm{b}}}}{r_{n}^{-\eta}} \mu_{\mathrm{b}} t\right.\right. \\
& \left.\left.-\sum_{k \notin \mathcal{K}} \frac{r_{b_{0}, b_{k}}^{-\eta_{\mathrm{B}}}}{r_{n}^{-\eta}} \mu_{\mathrm{B}} t\right)\right] \\
& =\mathbb{E}_{\Phi_{\mathrm{u}}}\left[\prod_{j=N}^{\infty} \exp \left(-\frac{r_{b_{0}, u_{j}}^{-\eta}}{r_{n}^{-\eta}} t\right)\right] \\
& \cdot \mathbb{E}_{\Phi_{\mathrm{b}}}\left[\prod_{k \in \mathcal{K}} \exp \left(-\frac{r_{b_{0}, b_{k}}^{-\eta_{\mathrm{b}}}}{r_{n}^{-\eta}} \mu_{\mathrm{b}} t\right) \prod_{k \notin \mathcal{K}} \exp \left(-\frac{r_{b_{0}, b_{k}}^{-\eta_{\mathrm{B}}}}{r_{n}^{-\eta}} \mu_{\mathrm{B}} t\right)\right] \\
& =\exp \left(-2 \pi \lambda_{\mathrm{u}} \int_{r_{N-1}}^{\infty}\left(1-e^{-r^{-\eta} r_{n}^{\eta} t}\right) r \mathrm{~d} r\right) \\
& \cdot \exp \left(-2 \pi \lambda_{\mathrm{b}} \int_{r_{b_{0}, b_{1}}>\kappa R_{\mathrm{c}}}^{R_{\mathrm{c}}}\left(1-e^{-r^{-\eta_{\mathrm{b}}} r_{n}^{\eta} \mu_{\mathrm{b}} t}\right) r \mathrm{~d} r\right) \\
& \cdot \exp \left(-2 \pi \lambda_{\mathrm{b}} \int_{R_{\mathrm{c}}}^{\infty}\left(1-e^{-r^{-\eta_{\mathrm{B}}} r_{n}^{\eta} \mu_{\mathrm{B}} t}\right) r \mathrm{~d} r\right)
\end{aligned}
$$

$$
\begin{gathered}
=\exp \left(\pi N \lambda_{\mathrm{b}} r_{N-1}^{2}+\frac{2 \pi N \lambda_{\mathrm{b}}}{\eta} r_{n}^{2} t^{\frac{2}{\eta}} \bar{\Gamma}\left(-\frac{2}{\eta}, \frac{r_{n}^{\eta}}{r_{N-1}^{\eta}} t\right)\right) \\
\cdot \exp \left(\pi \lambda_{\mathrm{b}} \kappa^{2} R_{\mathrm{c}}^{2}+\frac{2 \pi \lambda_{\mathrm{b}}}{\eta_{\mathrm{b}}}\left(R_{\mathrm{c}}^{2} E_{\frac{\eta_{\mathrm{b}}+2}{\eta_{\mathrm{b}}}}\left(\frac{r_{n}^{\eta}}{R_{\mathrm{c}}^{\eta_{\mathrm{b}}}} \mu_{\mathrm{b}} t\right)\right.\right. \\
\left.\left.-r_{b_{0}, b_{1}}^{2} E_{\frac{\eta_{\mathrm{b}}+2}{\eta_{\mathrm{b}}}}\left(\frac{r_{n}^{\eta}}{\left(\kappa R_{\mathrm{c}}\right)_{\mathrm{b}}} \mu_{\mathrm{b}} t\right)\right)\right) \\
\cdot \exp \left(\frac{2 \pi \lambda_{\mathrm{b}}}{\eta_{\mathrm{B}}}\left(r_{n}^{\eta} \mu_{\mathrm{B}} t\right)^{\frac{2}{\eta_{\mathrm{B}}}} \bar{\Gamma}\left(-\frac{2}{\eta_{\mathrm{B}}}, \frac{r_{n}^{\eta}}{R_{\mathrm{c}}^{\eta_{\mathrm{B}}}} \mu_{\mathrm{B}} t\right)\right) \cdot
\end{gathered}
$$

Putting (78) into (73) with $a_{n}=r_{n} / r_{N-1}$, we obtain $F_{\bar{\rho}_{0, n} \mid r_{n}, a_{n}}(\gamma)$ as in (32).

\section{APPENDIX D \\ PROOF OF PROPOSITION 3}

The user spectral efficiency averaged over all geometries in a full-duplex network is

$$
\begin{aligned}
\overrightarrow{\vec{C}} & =\mathbb{E}\left[\vec{C}\left(\vec{\rho}_{0}\right)\right] \\
& =\mathbb{E}\left[\mathbb{E}\left[\log _{2}\left(1+\overrightarrow{\mathrm{SIR}}_{0} \mid \vec{\rho}_{0}\right)\right]\right] \\
& =\mathbb{E}\left[\int_{0}^{\infty} \mathbb{P}\left[\log _{2}\left(1+\overrightarrow{\mathrm{SIR}}_{0} \mid \vec{\rho}_{0}\right)>\gamma^{\prime}\right] \mathrm{d} \gamma^{\prime}\right] \\
& =\mathbb{E}\left[\int_{0}^{\infty} \frac{\log _{2} e}{\gamma+1}\left(1-F_{\overrightarrow{\mathrm{SIR}}_{0} \mid \vec{\rho}_{0}}(\gamma)\right) \mathrm{d} \gamma\right] \\
& =\int_{0}^{\infty} \frac{\log _{2} e}{\gamma+1}\left(1-\mathbb{E}\left[F_{\overrightarrow{\operatorname{SIR}}_{0} \mid \vec{\rho}_{0}}(\gamma)\right]\right) \mathrm{d} \gamma \\
& =\int_{0}^{\infty} \frac{\log _{2} e}{\gamma+1} \mathbb{E}\left[e^{-\gamma / \vec{\rho}_{0}}\right] \mathrm{d} \gamma
\end{aligned}
$$

where the outer and inner expectations in (80) are over $\vec{\rho}_{0}$ and over the fading, respectively, while (82) follows from the variable change $\gamma^{\prime}=\log _{2}(1+\gamma)$. Invoking (72) into (84) and then averaging the resulting expression using the density function in (8) (with $n=0$ and $\lambda=\lambda_{\mathrm{b}}$ ), we arrive at (42).

\section{APPENDIX E}

PROOF OF PROPOSITION 4

The per-BS spectral efficiency averaged over all geometries in a full-duplex network is

$$
\begin{aligned}
\overleftarrow{\bar{C}} & =\sum_{n=0}^{N-1} \mathbb{E}\left[\overleftarrow{C}\left(\overleftarrow{\rho}_{0, n}\right)\right] \\
& =\sum_{n=0}^{N-1} \int_{0}^{\infty} \frac{\log _{2} e}{\gamma+1}\left(1-\mathbb{E}\left[F_{\overleftarrow{S I R}_{0, n} \mid \bar{\rho}_{0, n}}(\gamma)\right]\right) \mathrm{d} \gamma \\
& =\sum_{n=0}^{N-1} \int_{0}^{\infty} \frac{\log _{2} e}{\gamma+1} \mathbb{E}\left[e^{-\gamma / \bar{\rho}_{0, n}}\right] \mathrm{d} \gamma \\
& =\sum_{n=0}^{N-1} \int_{0}^{\infty} \frac{\log _{2} e}{\gamma+1} \mathbb{E}\left[\mathbb{E}\left[e^{-\gamma / \bar{\rho}_{0, n}} \mid r_{n}, a_{n}\right]\right] \mathrm{d} \gamma .
\end{aligned}
$$

Plugging (78) into (88) and then averaging the resulting expression by means of the PDF in (10) with $\lambda=N \lambda_{\mathrm{b}}$, we obtain the result in (48). 


\section{REFERENCES}

[1] M. Duarte and A. Sabharwal, "Full-duplex wireless communications using off-the-shelf radios: Feasibility and first results," in Proc. Annual Asilomar Conf. Signals, Syst., Comp., Pacific Grove, CA, Nov. 2010, pp. $1558-1562$.

[2] J. I. Choi, M. Jain, K. Srinivasan, P. Levis, and S. Katti, "Achieving single channel, full duplex wireless communication," in Proc. ACM int. conf. on Mobile comp. and net., Sept. 2010, pp. 1-12.

[3] M. Jain, J. I. Choi, T. Kim, D. Bharadia, S. Seth, K. Srinivasan, P. Levis, S. Katti, and P. Sinha, "Practical, real-time, full duplex wireless," in Proc. ACM int. conf. on Mobile comp. and net., Sept. 2011, pp. 301-312.

[4] D. Bharadia, E. McMilin, and S. Katti, "Full duplex radios," in Proc. ACM SIGCOMM, Aug. 2013, vol. 43, pp. 375-386.

[5] S. Hong, J. Brand, J. Choi, M. Jain, J. Mehlman, S. Katti, and P. Levis, "Applications of self-interference cancellation in 5G and beyond," IEEE Commun. Mag., vol. 52, no. 2, pp. 114-121, Feb. 2014

[6] Z. Zhang, X. Chai, K. Long, A. V. Vasilakos, and L. Hanzo, "Full duplex techniques for 5G networks: self-interference cancellation, protocol design, and relay selection," IEEE Commun. Mag., vol. 53, no. 5, pp. 128-137, May 2015.

[7] X. Zhang, W. Cheng, and H. Zhang, "Full-Duplex transmission in PHY and MAC layers for 5G mobile wireless networks," IEEE Wireless Commun. Mag., vol. 22, no. 5, pp. 112-121, Oct. 2015.

[8] D. Kim, H. Lee, and D. Hong, "A survey of in-band full-duplex transmission: From the perspective of PHY and MAC layers," IEEE Commun. Surveys Tuts., vol. 17, no. 4, pp. 2017-2046, FourthQuarter 2015.

[9] D. Bharadia and S. Katti, "Full duplex MIMO radios," in Proc. 11th USENIX Symposium on Networked Systems Design and Implementation, Apr. 2014, pp. 359-372.

[10] Z. Tong and M. Haenggi, "Throughput analysis for wireless networks with full-duplex radios," in Proc. IEEE Wireless Commun. and Networking Conf., Mar. 2015.

[11] M. Mohammadi, H. A. Suraweera, Y. Cao, I. Krikidis, and C. Tellambura, "Full-duplex radio for uplink/downlink wireless access with spatially random nodes," IEEE Trans. Commun., vol. 63, no. 12, pp. 5250-5266, Dec. 2015.

[12] C. Psomas and I. Krikidis, "Outage analysis of full-duplex architectures in cellular networks," in Proc. IEEE Veh. Technol. Conf., May 2015.

[13] S. Goyal, P. Liu, S. Hua, and S. Panwar, "Analyzing a full-duplex cellular system," in Proc. Conf. Inform. Sciences Syst. (CISS), Mar. 2013, pp. $1-6$.

[14] M. S. Sim, M. Chung, D. Kim, J. Chung, D. K. Kim, and C.-B. Chae, "Nonlinear self-interference cancellation for full-duplex radios: From link- and system-level performance perspectives," Available online: https://arxiv.org/abs/1607.01912/, 2016.

[15] J. Lee and T. Q. S. Quek, "Hybrid full-/half-duplex system analysis in heterogeneous wireless networks," IEEE Trans. Wireless Commun., vol. 15, no. 5, pp. 2883-2895, May 2015.

[16] A. Lozano and N. Jindal, "Are yesterday's information-theoretic fading models and performance metrics adequate for the analysis of today's wireless systems?," IEEE Commun. Mag., vol. 50, no. 11, pp. 210-217, Nov. 2012.

[17] R. K. Mungara and A. Lozano, "Interference surge in full-duplex wireless systems," in Proc. Annual Asilomar Conf. Signals, Syst., Comp., Nov. 2015, pp. 25-29.

[18] G. Caire, N. Jindal, M. Kobayashi, and N. Ravindran, "Multiuser MIMO achievable rates with downlink training and channel state feedback," IEEE Trans. Inform. Theory, vol. 56, no. 6, pp. 2845-2866, June 2010.

[19] A. AlAmmouri, H. ElSawy, O. Amin, and M. S. Alouini, "Inband full-duplex communications for cellular networks with partial uplink/downlink overlap," in Proc. IEEE Global Commun. Conf., Dec. 2015, pp. 1-7.

[20] T. D. Novlan, H. S. Dhillon, and J. G. Andrews, "Analytical modeling of uplink cellular networks," IEEE Trans. Commun., vol. 12, no. 6, pp. 2669-2679, June 2013.

[21] J. G. Andrews, A. K. Gupta, and H. S. Dhillon, "A primer on cellular network analysis using stochastic geometry," Available online: https://arxiv.org/abs/1604.03183/, 2016.

[22] M. Haenggi, Stochastic Geometry for Wireless Networks, Cambridge Univ. Press, Cambridge, U. K., 2012.

[23] L. Choi and R. D. Murch, "A transmit preprocessing technique for multiuser MIMO systems using a decomposition approach," IEEE Trans. Wireless Commun., vol. 3, no. 1, pp. 20-24, Jan. 2004.
[24] T. Sarkar, Z. Ji, K. Kim, A. Medouri, and M. Salazar-Palma, "A survey of various propagation models for mobile communication," IEEE Antennas Propagat. Mag., vol. 45, no. 3, pp. 51-82, June 2003.

[25] A. F. Molisch, "A generic model for mimo wireless propagation channels in macro- and microcells," IEEE Trans. Signal Processing, vol. 52, no. 1, pp. 61-71, Jan. 2004.

[26] 3GPP TR 36.828 V11.0.0, "Further Enhancements to LTE Time Division Duplex (TDD) for Downlink-Uplink (DL-UL) Interference Management and Traffic Adaptation," Tech. Rep., 3rd Generation Partnership Project 3GPP, www.3gpp.org, June 2012.

[27] M. Haenggi, "On distances in uniformly random networks," IEEE Trans. Inform. Theory, vol. 51, no. 10, pp. 3584-3586, Oct. 2005.

[28] F. J. Martín-Vega, F. J. López-Martínez, G. Gomez, and M. C. AguayoTorres, "Multi-user coverage probability of uplink cellular systems: A stochastic geometry approach," in Proc. IEEE Global Commun. Conf., Dec. 2014, pp. 3989-3994.

[29] R. K. Mungara, D. Morales-Jiménez, and A. Lozano, "System-level performance of interference alignment," IEEE Trans. Wireless Commun., vol. 14, no. 2, pp. 1060-1070, Feb. 2015.

[30] G. George, R. K. Mungara, and A. Lozano, "An analytical framework for Device-to-Device communication in cellular networks," IEEE Trans. Wireless Commun., vol. 14, no. 11, pp. 6297-6310, Nov. 2015.

[31] G. George, R. K. Mungara, A. Lozano, and M. Haenggi., "Ergodic spectral efficiency in MIMO cellular networks," IEEE Trans. Wireless Commun., to appear, 2017.

[32] C. C. Chan and S. V. Hanly, "Calculating the outage probability in a CDMA network with spatial poisson traffic," IEEE Trans. Veh. Technol., vol. 50, no. 1, pp. 183-204, Jan. 2001.

[33] A. Ghasemi and E. S. Sousa, "Interference aggregation in spectrumsensing cognitive wireless networks," IEEE J. Select. Topics Signal Processing, vol. 2, no. 1, pp. 41-56, Feb. 2008.

[34] J. Guo, S. Durrani, and X. Zhou, "Outage probability in arbitrarilyshaped finite wireless networks," IEEE Trans. Commun., vol. 62, no. 2 pp. 699-712, Feb. 2014.

[35] B. Blaszczyszyn and M. Karray, "Spatial distribution of the SINR in Poisson cellular networks with sector antennas," IEEE Trans. Wireless Commun., vol. 15, no. 1, pp. 581-593, Jan. 2016.

[36] J. Abate and W. Whitt, "Numerical inversion of Laplace transforms of probability distributions," ORSA J. Compt., vol. 7, no. 1, pp. 36-43, 1995.

[37] C. A. O'cinneide, "Euler summation for Fourier series and Laplace transform inversion," Commun. Statist. -Stochastic Models, vol. 13, no. 2, pp. 315-337, 1997.

[38] W. C. Y. Lee, "Estimate of channel capacity in rayleigh fading environments," IEEE Trans. Veh. Technol., vol. 39, no. 3, pp. 187-189, Aug. 1990.

[39] L. Ozarow, S. Shamai, and A. D. Wyner, "Information theoretic considerations for cellular mobile radio," IEEE Trans. Veh. Technol., vol. 43, no. 2, pp. 359-378, May 1994.

[40] S. Catreux, P. F. Driessen, and L. J. Greenstein, "Data throughputs using multiple-input multiple-output (MIMO) techniques in a noise-limited cellular environment," IEEE Trans. Wireless Commun., vol. 1, no. 2, pp. 226-235, Apr. 2002.

[41] I. S. Gradshteyn and I. M. Ryzhik, Table of Integrals, Series, and Products, Academic Press, San Diego, 7th edition, 2007.

[42] H. Elshaer, F. Boccardi, M. Dohler, and R. Irmer, "Downlink and uplink decoupling: A disruptive architectural design for 5G networks," in Proc. IEEE Global Commun. Conf., Dec. 2014, pp. 1798-1803.

[43] A. Lozano and D. C. Cox, "Integrated dynamic channel assignment and power control in TDMA mobile wireless communication systems," IEEE J. Select. Areas Commun., vol. 17, pp. 2031-2040, Nov. 1999.

[44] X. Wu, S. Tavildar, S. Shakkottai, T. Richardson, J. Li, R. Laroia, and A. Jovicic, "FlashLinQ: A synchronous distributed scheduler for peerto-peer ad hoc networks," IEEE/ACM Trans. Networking, vol. 21, no. 4, pp. 1215-1228, Aug. 2013.

[45] N. Naderializadeh and A. S. Avestimehr, "ITLinQ: A new approach for spectrum sharing in Device-to-Device communication systems," IEEE J. Select. Areas Commun., vol. 32, no. 6, pp. 1139-1151, June 2014.

[46] R. K. Mungara, X. Zhang, A. Lozano, and R. W. Heath Jr., "Analytical characterization of ITLinQ: Channel allocation for Device-to-Device communication networks," IEEE Trans. Wireless Commun., vol. 15, no. 5, pp. 3603-3615, May 2016.

[47] X. Yi and G. Caire, "Optimality of treating interference as noise: A combinatorial perspective," IEEE Trans. Inform. Theory, vol. 62, no. 8, pp. 4654-4673, Aug. 2016. 
[48] J. M. B. da Silva, G. Fodor, and C. Fischione, "Spectral efficient and fair user pairing for full-duplex communication in cellular networks," IEEE Trans. Wireless Commun., vol. 15, no. 11, pp. 7578-7593, Nov. 2016.

[49] Y.-S. Choi and H. Shirani-Mehr, "Simultaneous transmission and reception: Algorithm, design and system level performance," IEEE Trans. Wireless Commun., vol. 12, no. 12, pp. 5992-6010, Dec. 2013.

[50] O. Simeone, E. Erkip, and S. Shamai, "Full-duplex cloud radio access networks: An Information-Theoretic viewpoint," IEEE Wireless Commun. Lett., vol. 3, no. 4, pp. 413-416, Aug. 2014.

[51] S. A. Jafar, Interference Alignment: A New Look at Signal Dimensions in a Communication Network, vol. 7, Foundations and Trends in Communications and Information Theory, 2011.

[52] J. G. Andrews, S. Buzzi, W. Choi, S. Hanly, A. Lozano, A. C. K. Soong, and J. C. Zhang, "What will 5G be?," IEEE J. Select. Areas Commun., vol. 32, no. 6, pp. 1065-1082, June 2014.

[53] F. Boccardi, R. W. Heath Jr., A. Lozano, T. Marzetta, and P. Popovski, "Five disruptive technology directions for 5G," IEEE Commun. Mag., vol. 52, no. 2, pp. 74-80, Feb. 2014.

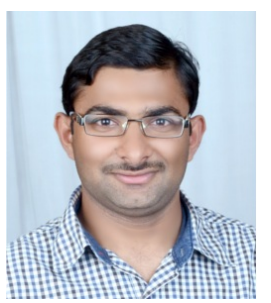

Ratheesh K. Mungara (S'13 - M'17) received the B.Tech. degree in electronics and communications engineering from Jawaharlal Nehru Technological University, Hyderabad, India, in 2005, the M.Tech. degree in communication systems from the National Institute of Technology, Tiruchirappalli, India, in 2007, and the Ph.D. degree in information and communication technologies from Universitat Pompeu Fabra (UPF), Barcelona, Spain, in 2016. He is currently working as a postdoctoral researcher at UPF. His general research interests are modeling and analysis of wireless networks using stochastic geometry tools. His current focus is on interference management techniques, device-to-device communication, and full-duplex communication in cellular networks.

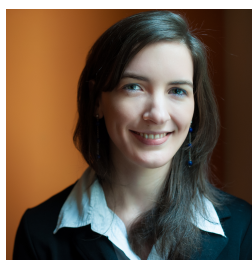

Ilaria Thibault (S'10 - M'13) received her B.S. and M.Sc. degrees in telecommunications engineering from the University of Bologna, Italy, in 2006 and 2009 , respectively, and her Ph.D. degree in information and communication technologies jointly from the University of Bologna, Italy, and from Pompeu Fabra University, Barcelona, Spain, in 2013. She is currently working as principal researcher in Vodafone Group R\&D and focusing on the development of $5 \mathrm{G}$ technologies and connected car solutions.

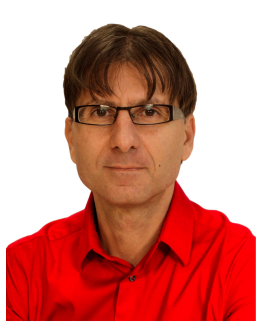

Angel Lozano (S'90 - M'99 - SM'01 - F'14) received the M.Sc. and Ph.D. degrees in electrical engineering from Stanford University, Stanford, CA, in 1994 and 1998, respectively. In 1999, he joined Bell Labs (Lucent Technologies, now Nokia) in Holmdel, NJ, where he was a Member of the Wireless Communications Research Department until 2008. Between 2005 and 2008, he was also an Adj. Associate Professor of Electrical Engineering at Columbia University, New York, NY. He is currently a Professor of Information and Communication Technologies at Universitat Pompeu Fabra (UPF), Barcelona, Spain. He has published extensively, holds 15 patents, and has contributed to several books. $\mathrm{He}$ has been an Associate Editor of the IEEE Transactions on Information Theory (2011-2014) and the IEEE Transactions on Communications (19992009), as well as a Guest Editor for various special issues. He received the 2009 Stephen O. Rice Prize for the Best Paper published in the IEEE Transactions on Communications, the 2016 Fred W. Ellersick prize to the best paper published in the IEEE Communications Magazine, and the 2016 Communications Society \& Information Theory Society joint paper award. 